\title{
Characterisation of the Ubiquitin-ESCRT pathway in Asgard archaea sheds new light on origins of membrane trafficking in eukaryotes
}

Tomoyuki Hatano ${ }^{1}$, Saravanan Palani ${ }^{1 *}$, Dimitra Papatziamou ${ }^{2 *}$, Diorge P. Souza ${ }^{3 *}$, Ralf Salzer ${ }^{3 *}$, Daniel Tamaritt, ${ }^{4 *}$ Mehul Makwana ${ }^{2}$, Antonia Potter ${ }^{2}$, Alexandra Haig ${ }^{2}$, Wenjue $\mathrm{Xu}^{2}$, David Townsend ${ }^{6}$, David Rochester ${ }^{6}$, Dom Bellini ${ }^{3}$, Hamdi M. A. Hussain ${ }^{1}$, Thijs Ettema ${ }^{4}$, Jan Löwe ${ }^{3}$, Buzz Baum $^{3 \S}$, Nicholas P. Robinson ${ }^{2 \S}$, Mohan Balasubramanian ${ }^{1 \S}$

${ }^{1}$ Centre for Mechanochemical Cell Biology, Division of Biomedical Sciences, Warwick Medical School, University of Warwick, Coventry CV4 7AL, United Kingdom

${ }^{2}$ Division of Biomedical and Life Sciences, Faculty of Health and Medicine, Lancaster University, Lancaster LA1 4YG, United Kingdom

${ }^{3}$ MRC Laboratory of Molecular Biology, Cambridge CB2 OQH, United Kingdom

${ }^{4}$ Laboratory of Microbiology, Department of Agrotechnology and Food Sciences, Wageningen University, Wageningen 6708 WE, the Netherlands

${ }^{5}$ Department of Aquatic Sciences and Assessment, Swedish University of Agricultural Sciences, SE75007 Uppsala, Sweden

${ }^{6}$ Department of Chemistry, Lancaster University, Lancaster LA1 4YB, United Kingdom

*These authors contributed equally to this work and are listed alphabetically

§ Correspondence to Mohan Balasubramanian- M.K.Balasubramanian@warwick.ac.uk; Nick Robinson- n.robinson2@lancaster.ac.uk ; Buzz Baum- bbaum@mrc-Imb.cam.ac.uk 


\section{SUMMARY}

The ESCRT machinery performs a critical role in membrane remodelling events in all eukaryotic cells, including in membrane trafficking, membrane repair, cytokinetic abscission, in viral egress, and in the generation of extracellular vesicles. While the machinery is complex in modern day eukaryotes, where it comprises dozens of proteins, the system has simpler and more ancient origins. Indeed, homologues of ESCRT-III and the Vps4 ATPase, the proteins that execute the final membrane scission reaction, play analogous roles in cytokinesis and potentially in extracellular vesicle formation in TACK archaea where ESCRT-I and II homologues seem to be absent. Here, we explore the phylogeny, structure, and biochemistry of homologues of the ESCRT machinery and the associated ubiquitylation system found in genome assemblies of the recently discovered Asgard archaea. In these closest living prokaryotic relatives of eukaryotes, we provide evidence for the ESCRT-I and II sub-complexes being involved in the ubiquitin-directed recruitment of ESCRT-III, as it is in eukaryotes. This analysis suggests a preeukaryotic origin for the Ub-coupled ESCRT system and a likely path of ESCRT evolution via a series of gene duplication and diversification events.

\section{INTRODUCTION}

The ESCRT (Endosomal Sorting Complex Required for Transport) machinery is composed of several protein complexes and associated accessory proteins, including the ESCRT-0, -I, -II, -III subcomplexes, Vps4, and ALIX/Bro1 ${ }^{1-18}$ (Figure S1). These proteins act in sequence to bind, deform and cut membranes during membrane trafficking ${ }^{2-5}$, cell division ${ }^{19,20}$, viral egress ${ }^{21,22}$ and in other important topologically similar membrane remodelling events in eukaryotes ${ }^{23-39}$. When driving the formation of multivesicular bodies (MVBs), where the role of ESCRT in trafficking has been best characterised, the ESCRT machinery is recruited to endosomal membranes by ubiquitylated transmembrane proteins that are targeted to vesicles ${ }^{40}$. The ubiquitin $(\mathrm{Ub})$ moiety is recognised by ESCRT-0 and $-I$ subcomplexes, which bind the ubiquitin $\beta$-grasp fold ${ }^{9,14,41,42}$. ESCRT-I proteins together with the ESCRT-II sub-complex then corral these ubiquitylated transmembrane proteins into membrane domains ${ }^{9,11}$. Finally, the ESCRT-II sub-complex nucleates the local formation of ESCRT-III co-polymers which, through action of the Vps4 ATPase, undergo structural changes to drive membrane invagination and scission to release vesicles containing the Ub-marked transmembrane proteins ${ }^{43-53}$.

ESCRT-I, -II, -III components are found conserved across the eukaryotic lineages, pointing to this machinery being present in the last eukaryotic common ancestor (LECA; Note ESCRT-0 is only encountered in Opisthokonta) ${ }^{54}$. Homologues of ESCRT-III and Vps4 are coded by the genomes of many archaeal species ${ }^{55-60}$, and shown to function in archaeal membrane remodelling during cytokinesis and virus release. More recently, PspA and Vipp1 have been recognized as bacterial ESCRT-III related proteins $^{61-63}$. These observations suggest that a subset of ESCRT components have more ancient evolutionary origins. Furthermore, the identification of a full complement of ubiquitin and its activating enzymes (E1, E2, and E3) in some archaeal species has provided evidence for ubiquitylation cascades functioning in protein degradation in the archaeal ancestors of eukaryotes ${ }^{58,64,65}$.

This begs the question: when in evolution did ESCRT-I and ESCRT-II machineries arise, and when was ubiquitylation co-opted to regulate ESCRT? Metagenome assemblies of the recently discovered Asgard archaea, the closest living relatives of eukaryotes, have revealed that homologues of the entire ubiquitylation cascade, ESCRT-III (and Vps4), and components of the ESCRT-I and ESCRT-II subcomplexes are all encoded by the genomes of these archaea ${ }^{57,58}$. However, validating this conclusion in cells is currently very difficult, as only one Asgard member has been isolated and cultured $^{66}$; and its growth rate, physiology and the lack of essential tools prevent its use as a cell biological model.

To circumvent these challenges, here we apply a diverse set of experimental approaches to characterise Asgard archaeal homologues of the eukaryotic Ub-ESCRT system, focusing on the ESCRT-I and 
ESCRT-II subcomplexes. Our analysis shows that, like its eukaryotic counterparts, the Asgard ESCRTI subcomplex stably recognises ubiquitin. Furthermore, by carrying out a comprehensive two-hybrid analysis, we have been able to identify protein-protein interactions within and between the different ESCRT subcomplexes. Additionally, our data show that Asgard ESCRT subcomplexes have likely arisen through a process of gene duplication and diversification, prior to the evolution of more complex eukaryotic ESCRT assemblies. Taken together, this work reveals the presence of a genetically streamlined ubiquitin-associated multi-component ESCRT pathway that predates the emergence of the eukaryotic ESCRT machinery.

\section{RESULTS}

\section{Phylogenetic analyses indicate that Asgard archaeal genomes encode homologues of most, but not all components of the Ub-ESCRT machinery}

Many of the recently discovered Asgard archaeal genomes encode a wide array of so-called 'eukaryotic signature proteins' (ESPs) and appear unique amongst prokaryotes in possessing close homologues of most of the proteins that make up the ESCRT-I and -II complexes, together with ESCRT-III and Vps4 and homologues of ubiquitin and the associated ubiquitin-modification enzymes ${ }^{57,58}$. While these data suggest the possibility of some Asgard archaea possessing functional Ub-ESCRT membrane trafficking machinery, it is noticeable that Asgard genomes appear to lack a number of genes encoding proteins essential for ESCRT function in eukaryotes (Figure 1A). Thus, to put this hypothesis to the test, we began by generating a catalogue of proteins with homology to the components of the eukaryotic UbESCRT pathway in distinct Asgard phyla shown in Figure 1A and Figure S2 and Figure S3.

We focused on Asgard archaea that have metagenomic assemblies that are near complete based on them being assigned to small numbers of contigs and on estimates of genome coverage ${ }^{57,58}$. Within such genomes, as previously described ${ }^{57,58}$, we were able to identify genes coding for close homologues of ubiquitin, ubiquitin modifying enzymes, ESCRT-I components, ESCRT-II subunits, together with homologues of ESCRT-III and Vps4 (Figure 1A and Figure S3) ${ }^{58,67}$. These analyses strongly suggest that many Asgard archaea possess a bona fide eukaryote-like ESCRT-system.

Since gene clustering in prokaryotes frequently brings together genes with common functions, we sought to determine the extent to which ubiquitylation and ESCRT related genes are found co-located within specific regions of Asgard genomes (Figure 1B and C). As previously described ${ }^{58}$ within a single Odinarchaeota genome the full set of putative gene-products with homology to Ub-ESCRT were found together within a single gene cluster (Figure 1C). We extended this analysis by developing a simple metric of gene clustering, which was applied to Heimdall-, Loki-, Thor, and the recently described Helarchaeotal genomes, all which harbour ESCRT genes ${ }^{57,58,67}$. This was achieved by measuring the fraction of genomes within each phylum in which each pair of genes co-localises within less than $10 \mathrm{~kb}$ (Figure 1B; white indicates no evidence of co-location and deep purple indicates full co-location) ${ }^{68}$. This analysis revealed cases where the entire set of genes was clustered together in genomes Hel and Heimdall archaea genomes, and was found organised into two relatively discrete Ub and ESCRT genomic regions in Lokiarchaeota (Figure 1B). In addition, we observed a consistent pattern of association across genomes in which the genes for ESCRT-III and Vps4 were found most tightly associated with homologues of $\mathrm{Vps} 25$ (Figure 1B-C, S3). This is striking as Vps25 is the subunit of the ESCRT-II complex in eukaryotes that recruits ESCRT-III to membranes, triggering vesicle budding ${ }^{1-18}$. Vps22/Vps36 homologues (ESCRT-II components) were found to have a similar but slightly less consistent pattern of co-location with ESCRT-III and Vps4, and the gene was usually closely associated with Vps25 (Figure 1B-C, S3).

During this analysis, we also noted that, whereas eukaryotic Vps22 and Vps25 function as part of a single hetero-tetrameric complex together with a divergent Vps22 homologue, Vps36, Asgard archaeal genomes possessed a single gene encoding a Vps22/Vps36-like protein (Figure $1 \mathrm{~A}$ and $\mathrm{C}$ ). $\mathrm{A}$ 
phylogenetic analysis was used to confirm that the Asgard Vps22-like protein is a closer homologue of Vps22 than it is of Vps36 (Figure 1D, Figure S4). This suggests that the eukaryotic-specific protein, Vps36, arose from a Vps22Nps36 homolog after the divergence of Vps22 and Vps25 in Asgard archaea.

Asgard genomes also code for clear homologues of the eukaryotic ESCRT-I machinery, including homologues of Vps23 (which contain a Ubiquitin E2-Variant or "UEV" domain) and Vps28, both containing steadiness box (SB) domains. Interestingly, these genes were not tightly clustered in Asgard genomes (Figure 1B-C, Figure S5). Furthermore, the organisation of this set of genes was variable across lineages; including genomes in which individual domains were brought together to form fusion proteins (Figure 1B-C, Figure S5-8). In addition, Asgard archaea were found to lack a clear homologue of the eukaryotic Vps37. This ESCRT-I subunit is a homologue of Vps23 (reference 68 and Figure S58), implying that Vps37 may have arisen later in evolution in the branch leading to eukaryotes. In summary, with the notable exception of the Thorarchaeota, which seemingly lack true ubiquitin homologues (Figure 1A-C and Figure S5), most Asgard genomes have the potential to encode proteins that together resembles large parts of the conserved eukaryotic Ub-ESCRT system.

\section{Asgard archaeal ESCRT-I subcomplexes bind ubiquitin}

In eukaryotes, a variant of the ubiquitin E2 (UEV) domain plays a key role in Ub recognition by ESCRT${ }^{69}$. The UEV domain is similar in structure to the E2 region of the ubiquitin conjugating E2 enzymes, but lacks the key catalytic cysteine. We identified a number of similar proteins in Asgard archaea. The Heimdallarchaeota AB125 genome codes for four E2-like candidates: HeimAB125_07740, HeimAB125_09840, HeimAB125_11700 and HeimAB125_14070 (Figure S7A-B). Structural models of these Heimdall proteins were generated using I-TASSER ${ }^{-70-72}$ (Figure S7A) and structural superimposition used to confirm that, while two of the Heimdall proteins (HeimAB125_07740 and HeimAB125_09840) possess putative catalytic Cys residues (characteristic of bona fide Ẽ2 ubiquitinconjugating proteins), two (HeimAB125_11700 and HeimAB125_14070) did not contain cysteine residues at the expected catalytic positions (can we HIGHLIGHT this cysteine in Figure S6? - its hard to find!), raising the possibility that these may have UEV domains and function as ubiquitin-binding proteins (Figure S7A and B). One of these UEV domain-containing proteins represents a fusion of a Vps28 domain to UEV-Vps23 (containing ESCRT-I signatures: antiparallel coiled-coil stalk region and a steadiness box). This organisation raises the possibility that this Heimdallarchaeota protein harbours both Vps23-like ubiquitin-binding activity (via the UEV-domain) and Vps28-like functions, which are central to ESCRT-II subcomplex recruitment (Figure 2A-C and Figure S7 and S8). A phylogenetic analysis was used to confirm that both Asgard and eukaryotic UEV-like proteins cluster with eukaryotic Vps23 homologues and away from bona fide Ubiquitin E2 enzymes - suggesting a divergence in the structure and function of UEV and E2 domains that predates the Last Asgard and Eukaryotic Common Ancestor (LAsECA) (Figure 1E). Other Asgard species, most notably Odinarchaeota archaeon LCB_4, possess separable and distinct Vps23-like (containing the UEV domain) and Vps28 proteins, as seen in eukaryotes (Figure S8). Both these Odin proteins possess steadiness boxes, which in eukaryotes are critical for the assembly of the ESCRT-I subcomplexes ${ }^{9,73,74}$.

To test for physical interactions between these putative ESCRT-I proteins, we recombinantly expressed in E. coli and purified Heimdallarchaeota and Odinarchaeota ubiquitin together with their corresponding putative UEV-containing proteins and performed in vitro binding experiments. In the case of the Heimdallarchaeota proteins, the interaction between the purified full-length UEV-Vps23-Vps28 fusion protein and ubiquitin was analysed by chemical cross-linking followed by SDS-PAGE (Figure 2D). This revealed an increase in the apparent molecular weight of the protein upon ubiquitin binding and crosslinking (Figure 2D, top panel). In addition, we observed a ubiquitin-dependent increase in molecular weight for the isolated Heimdall UEV (Figure 2A, green), indicative of direct ubiquitin binding by this domain, rather than a different part of the full-length Vps23-Vps28 protein (Figure 2D, bottom panel). In 
eukaryotes, binding is mediated by an Ile-residue at position 44 (144) of Ubiquitin, which is part of a hydrophobic patch ${ }^{13}$. A model of Heimdall ubiquitin was superimposed on an available crystal structure of ubiquitin in a complex with UEV (Figure 2B-C) to identify the equivalent residues (V45 in Heimdall ubiquitin). When tested experimentally, the V45D mutation dramatically reduced the interaction between ubiquitin and the UEV domain (Figure 2D) - implying that this interaction resembles the one seen in eukaryotes.

The same was true of the equivalent Odinarchaeotal proteins. When Odin homologues of ubiquitin, Vps23 and Vps28 were purified and mixed in vitro, we observed the formation of a stable complex by size-exclusion chromatography (Figure 2E); mediated by an interaction between ubiquitin and the UEV domain-containing Vps23 (Figure 2E, S9, and S10A). Furthermore, when analysed by size-exclusion chromatography, Vps23 migrated through the column faster than expected for a monomer (Figure S9). Follow up size-exclusion chromatography coupled with multi-angle light scattering (SEC-MALS) analyses (Figure S10 A-C) revealed that the Vps23 protein forms a stable dimeric assembly (60.37 $\mathrm{kDa}$ ), while the calculated mass of the $\mathrm{Vps} 23-\mathrm{Vps} 28$ complex was consistent with a single subunit of Vps28 associating with the Vps23 dimer (yielding a combined molecular weight of $88.27 \mathrm{kDa}$ ). In this, the Odinarchaeotal Vps23-Vps28 complex appears like the eukaryotic ESCRT-I subcomplex, which assembles into a heterotrimeric core 'headpiece' comprised of Vps23, Vps28 and Vps37 ${ }^{74-76}$. As Asgard genomes encode Vps23-like proteins but lack their Vps37 homologues (Figure 1A), it is likely that the eukaryotic ESCRT-I (Fig. S8) arose from a heterotrimeric archaeal ESCRT-I subcomplex composed of a Vps23 homodimer and Vps28.

\section{Potential gene duplication and functional divergence of the eukaryotic ESCRT-II subcomplex from putative Asgard archaeal precursors.}

Turning to the ESCRT-II subcomplexes, our genomic analysis identified close homologues of Vps22 and Vps25 in Asgard archaeal genome assemblies, but not full-length Vps36 homologues. Since eukaryotic Vps22 and Vps36 are structurally related ${ }^{77,78}$, and form a Vps22-Vps36 heterodimer, we investigated whether Asgard Vps22 homologues might homodimerize. To test this idea, we used size exclusion chromatography (Figure 3A) and SEC-MALS (Figure 3B) to show that a Heimdall-Vps22 homologue (HeimAB125_14050) migrates with a size consistent with it forming a dimer. Moreover, after chemical crosslinking to stabilize the Vps22 complex, through the addition of BS3 (a homo-bifunctional cross-linker) or EDC (hetero-bifunctional cross-linker that generates zero-length isopeptide bonds), the Vps22 protein band detected using SDS-PAGE had a molecular weight of about $50 \mathrm{kDa}$, corresponding to a dimer (Figure 3C). Finally, using chemical cross-linking coupled with mass spectrometry (XL-MS), we identified two dimerization surfaces (41-47 and 160-167 amino acid regions) in the HeimAB125_14050 Vps22 homodimer (Figure 3D-E and Figure S11). Taken together, these data strongly suggest that Heimdallarcheota AB125 Vps22 forms a homodimer. Thus, it appears likely that the eukaryotic Vps22-Vps36 heterodimer arose during eukaryotic evolution following a gene duplication and diversification event - just as seems to have been the case for the ESCRT-I complex. It is notable, however, that when a SEC-MALS investigation was performed with the Odinarchaeota Vps22, we did not find evidence for its homodimerization. Since the Odin protein was observed to be monomeric (Figure S10D), it is currently unclear if it adopts a homodimeric architecture in the native host conditions or might instead form a heterodimer by interacting with as-yet undiscovered proteins.

Structural models for the Odinarchaeotal and Hemidallarchaeotal ESCRT-II subcomplex proteins using the I-TASSER ${ }^{70-72}$ and tr-Rosetta ${ }^{79}$ servers (Figure S12 A-B) suggested them having structural similarities with their eukaryotic counterparts, Vps22 and Vps25, based largely on the possession of shared tandem winged-helix $(\mathrm{WH})$ domains ${ }^{77,78,80}$. We examined the recombinant Odinarchaeota Vps22 and Vps25 by CD spectroscopy (Figure S12 C-F). This confirmed that both proteins are folded and have the predicted secondary structural elements. To further characterise Asgard ESCRT-II subunits, these proteins were submitted to crystallisation trials. In the case of Odinarchaeota Vps25, while we were unable to crystalise the full-length soluble protein, high quality crystals could be generated using an $\mathrm{N}$ - 
terminally truncated version (deleting $\mathrm{N}$-terminal residues 1 to 58 ), which were then used to determine the structure at a resolution of $1.80 \AA$ (Supplementary Table 1 and Figure 4A-C). Inspection of this structure revealed that the Asgard Vps 25 core is composed of a tandem WH domain repeat, consistent with the predicted tr-Rosetta model, and is very similar in structure to the equivalent eukaryotic protein (Figure 4C). Taken together, these findings provide further support for the idea that all the ESCRT-II subcomplex proteins in eukaryotes and Asgard archaea alike share a common molecular architecture based on a core tandem WH domain ${ }^{77,78}$. These data reinforce the concept that the ESCRT-II complex arose during archaeal and eukaryotic evolution through a series of gene duplication and specialization events $^{81,82}$.

\section{Key ESCRT complex protein-protein interactions revealed by yeast-2-hybrid analyses of the Asgard archaeal systems}

Since the ESCRT-I and ESCRT-II systems characterised above function as potential protein bridges that physically connect Ub-modified proteins with the ESCRT-III machinery, we wanted a systematic way to sensitively test for interactions within and across different subcomplexes. To do so, we carried out a comprehensive reciprocal yeast two hybrid analysis $(\mathrm{Y} 2 \mathrm{H})$ in budding yeast to identify pairwise protein interactions for the full set of Ub-ESCRT homologues from Thor-, Odin-, Loki- and Heimdallarchaeota (Figure 5A). As a control for this analysis, we used the same approach to systematically probe for interactions between proteins that are known to function as part of the ESCRT system in the fission yeast Schizosaccharomyces pombe (Figure S13 and Figure S14). Importantly, in these control experiments we were able to identify many of the expected interactions between components of the Ub-ESCRT system in fission yeast. This included the previously reported interactions within the respective ESCRT complexes; ESCRT-I (Sst6-Vps28), ESCRT-II (Vps22-Vps25, Vps36Vps25), ESCRT-III (Vps20-Vps32, Vps24-Did4), as well as published interactions that bridge the eukaryotic ESCRT-I and -II subcomplexes (Vps28-Vps36) and those that connect ESCRT-II and -III (Vps20-Vps25, Vps20-Vps22) (Figure S13A).

We then applied $\mathrm{Y} 2 \mathrm{H}$ analyses to systematically search for protein-protein interactions between ESCRT-related components encoded by Asgard archaeal genomes (Figure 5A). As expected, these assays revealed interactions between ESCRT-III components and the Vps4 ATPase. In line with the biochemical data presented above, these $\mathrm{Y} 2 \mathrm{H}$ analyses also identified interactions between ubiquitin and UEV domain-containing proteins in Heimdallarchaeota, Odinarchaeota and Lokiarchaeota (Figure $5 A$ and S14). Furthermore, the UEV domain-containing Vps23 homologues from Odinarchaeota and Heimdallarchaeota displayed interactions with Vps28, as expected if they formed an ESCRT-I subcomplex, whereas the Heimdallarchaeota utilise the single Vps23-Vps28 fusion protein homologue discussed above. The $\mathrm{Y} 2 \mathrm{H}$ assays also detected the interaction between ubiquitin and this Heimdall ESCRT-I fusion (Figure 5A and Figure S13B). Interestingly, this analysis also suggested an alternative pattern of protein interactions for the corresponding ESCRT-I proteins from Loki and Thor. In these two cases, the freestanding steadiness box protein, equivalent to the alpha-helical hairpin "headpiece ${ }^{, 73,74,83}$ coded within the same genomic neighbourhood as the rest of the Loki ESCRT machinery (Figure 1C), may come into play by interacting with Vps28 - mirroring the role of its eukaryotic counterpart in mediating interactions between Vps23, Vps28 and Vps37.

The $\mathrm{Y} 2 \mathrm{H}$ analyses also identified multiple interactions between the Lokiarchaeota and Heimdallarchaeota ESCRT-I (Vps28 and/or Vps23) and ESCRT-II (Vps22) complexes. In addition, the $\mathrm{Y} 2 \mathrm{H}$ experiments indicate that the ESCRT-II component Vps22 from Heimdall, Loki and Thor can interact with themselves, in agreement with the biochemical assays shown above. Finally, we identified numerous interactions linking the Asgard ESCRT-II and -III (Vps25-ESCRT-IIIB), Vps4-ESCRT-III and between the ESCRT-III homologues (-IIIA and -IIIB) (Figure 5A and Figure S14), and suggests the possibility of additional interactions between ESCRT-I and II subcomplexes with ESCRT-III proteins that bypass Vps25 in Loki and Thor archaeota. We note that although we were unable to detect many such interactions between the Odinarchaeotal proteins using the $\mathrm{Y} 2 \mathrm{H}$ approach, since Odin is a thermophile, 
300 it seems likely that the temperature used for these experiments $\left(25^{\circ} \mathrm{C}\right)$ may have influenced our ability to identify interactions between proteins that are optimised to fold and work at much higher physiological temperatures ${ }^{58}$.

\section{DISCUSSION:}

Here we provide experimental and computational support for the idea that many Asgard archaea possess a streamlined version of the Ub-ESCRT system present in eukaryotes (Figure 5B). It is clear from our analyses, however, that the precise composition differs between species and across phyla. This is especially the case for ESCRT-I subunit architecture. However, except for Thorarchaeota (in which ubiquitin encoding genes are yet to be identified), we found ubiquitin-binding UEV-domain containing proteins coded by the genomes analysed. While these domains were often harboured within proteins homologous to Vps23, which include a C-terminal alpha-helical headpiece region involved in ESCRT-I complex assembly, in other systems this alpha-helical 'steadiness box' domain was encoded by a freestanding protein. Alternative ESCRT-I domain arrangements were also observed, such as the UEV-Vps23-Vps28 fusion found in Heimdallarchaeota (Figure S8). Taken together, the clear synteny between genes of ubiquitylation apparatus and the ESCRT machinery in the Loki-, Hel-, Odin-, and Heimdallarchaeotal genomes, and the experimentally verified $\mathrm{Y} 2 \mathrm{H}$ interactions between ubiquitin and UEVs in all the Asgards investigated, support a model in which ubiquitylated substrates recruit the ESCRT-I in Asgard archaea.

In line with this, we were able to demonstrate direct binding between ubiquitin and UEV-containing proteins that was dependent on a conserved hydrophobic patch in ubiquitin. Although Asgard archaea appear to lack homologues of the eukaryotic ESCRT-I subunit Vps37 (which assembles into a trimer with Vps23 and Vps28), it is notable that Vps23 and Vps28 from Odinarchaeota assemble into a similar trimer that contains two copies of Vps23. It is therefore possible that all three proteins of the eukaryotic ESCRT-I complex evolved from a common ancestor containing an alpha-helical hairpin region, with Vps37 having arisen as a eukaryotic innovation ${ }^{73}$.

Similarly, the eukaryotic ESCRT-II subcomplex forms a 'Y-shaped' hetero-tetrameric structure consisting of a Vps22/Nps36 stalk which binds two Vps25 subunits ${ }^{77,78}$. Although Vps22 and Vps25 coding genes were readily identifiable in the Asgard genomes as reported previously, we were unable to identify Vps36 homologues. However, our biochemical and $\mathrm{Y} 2 \mathrm{H}$ interrogation suggest that Vps22 from several Asgard phyla likely forms homodimers. In the corresponding eukaryotic complex, all three of these ESCRT-II proteins (Vps22, Vps36 and Vps25) contain an evolutionarily conserved globular core consisting of tandem winged helix $(\mathrm{WH})$ domains ${ }^{77,78,80}$. The same appears true for the Asgard ESCRT-II machinery, based upon modelling of Vps22 and Vps25 proteins from Odinarchaeota, and the crystallographic Odinarchaeotal Vps25 structure. This suggests that all ESCRT-II proteins were initially derived from a single WH-domain protein progenitor, with Vps36 emerging during eukaryogenesis.

Do ESCRT-I, -II, and -III contribute to a single pathway? The colocation and synteny analyses strongly suggest this possibility. The colocation of ESCRT-III with Vps25 indicates that these proteins function in a related biochemical process. $\mathrm{Y} 2 \mathrm{H}$ experiments revealed consistent interactions between Vps 25 and ESCRT-IIIB. Furthermore, this analysis provided support for there being an interaction between ESCRTI and ESCRT-II. We also found an interaction between the Lokiarchaeota steadiness box protein and Vps22, supporting the existence of physical interactions between the ESCRT-I and ESCRT-II subcomplexes. These $\mathrm{Y} 2 \mathrm{H}$ data and the corresponding gene cluster analysis point to ESCRT -I, -II, and -III functioning in concert in Asgard archaea - although confirmation of this will require a future cell biological and/or biochemical analyses. While it is not yet clear how the Ub-ESCRT system evolved, we note that while ESCRT-III type proteins can be traced back to the last universal common ancestor $(\text { LUCA })^{63}$, the Vps4 and ESCRT-III pair can only be found in archaea, whereas Ub, ESCRT-I and ESCRT-II components are only found together in Asgard (with the exception of Thorarchaeota, which seemingly lack true ubiquitin homologues). This suggests a plausible pathway for the stepwise evolution 
353 of the eukaryotic ubiquitin-directed ESCRT-dependent membrane trafficking system as, from its simple beginnings in an archaeal progenitor, the machinery grew in complexity through successive rounds of domain concatenation, gene duplication and divergence (Figure 5B).

\section{MATERIALS AND METHODS}

\section{Genomic survey of protein homologues}

All genomes from organisms classified as Asgard archaea were downloaded from NCBI on December $5^{\text {th }}, 2020$. These genomes were taxonomically reclassified through a phylogenetic analysis based on a set of 15 ribosomal proteins encoded in co-locating genes ${ }^{84}$. To ensure annotation homogeneity, protein sequences were predicted de novo using Prodigal v2.6.3 $3^{85}$, and ribosomal protein genes were detected using psiblast ${ }^{86}$ using predetermined orthologous sequences ${ }^{58}$, aligned with Mafft-linsi v7.450 ${ }^{87}$ and processed with trimAl v1.4.rev $22^{88}$ to remove sites with over $50 \%$ gaps. All genomes containing at least 5 of these proteins were concatenated and used to reconstruct a tree with IQ-Tree $\mathrm{v} 2.0-\mathrm{rc} 1^{89}$ the LG+C60+R4+F model, using 1000 pseudoreplicates for ultrafast bootstrap ${ }^{90}$ and $\mathrm{SH}$-approximate likelihood ratio tests (Figure S1).

These reclassified genomes were then annotated using interproscan v5.48-83.091. A set of interpro domains were used as diagnostic for the inference of Vps23/37 (IPR017916), Vps28 (IPR007143, IPR037206), Vps23/37/28 (IPR037202), Vps22/36 (IPR016689, IPR040608, IPR021648), Vps25 (IPR008570, IPR014041), ESCRT-III (IPR005024), Vps4 (IPR007330, IPR031255, IPR015415), ubiquitin (IPR029071, IPR000626), ubiquitin-activating enzyme E1 (IPR000594), ubiquitin-conjugating enzyme E2 (IPR000608, IPR006575, IPR016135), ubiquitin-ligase enzyme E3 (IPR018611), and deubiquitinating enzyme (IPR000555) genes.

Co-location of these genes was investigated through custom perl scripts and visualised using $R$ ( $R$ Core Team 2018, R: A language and environment for statistical computing. R Foundation for Statistical Computing, Vienna, Austria. https://www.R-project.org/) and the packages ggplot2 ${ }^{92}$,cowplot (https://CRAN.R-project.org/package=cowplot), and genoPlotR ${ }^{93}$.

\section{Generation of model structures using I-TASSER server}

The model structures used in this study were generated as described below:

(1) The model structure of Heimdall E2-like proteins for Figure S7A were generated using I-TASSER ${ }^{70-}$ 72 (https://zhanglab.ccmb.med.umich.edu/l-TASSER/). No template structure was selected for this modelling. The amino acid sequence used for structural modelling is as follows; HeimAB125_07740 (27th-135th amino acid residues); (ii) HeimAB125_09840 (1st-107th amino acid residues); (iii) HeimAB125_14070 (51st-108th amino acid residues); (iv) HeimAB125_11700 (25th-122nd amino acid residues). The amino acid residues for the structural modelling were selected based on InterPro annotation of "UBIQUITIN_CONJUGAT_2". It is important to note that the c score of HeimAB125_14070 truncation was $\mathrm{NaN}$, due to lower reliability of the model structure.

(2) The model structure of Heimdall UEV domain in HeimAB125_14070 (1st-130th amino acid residues) in complex with ubiquitin (used in Figure 3B and S7B) was generated using I-TASSER with crystal structure of yeast UEV in complex with ubiquitin (PDB: 1UZXa).

(3) The model structure of Heimdall UEV domain in HeimAB125_14070 (1st-130th amino acid residues) for Figure S7A was generated by using I-TASSER with crystal structure of UEV (PDB: 1KPQ).

(4) The model structure of Heimdall ubiquitin (HeimAB125_14240) and Heimdall Vps22 (HeimAB125_14050) were generated by I-TASSER without assigning template structures.

In most of the cases, I-TASSER provided several models. We always used the model which showed the highest c scores. The score, templates and amino acid region were listed in Table S1.

\section{Asgard proteins used in this study}

The Heimdall-, Loki-, Odin-, and Thorarchaeota amino acid sequences were obtained from Uniprot (https://www.uniprot.org/) and the Uniprot Entry ID are listed in Table S2. The corresponding genes 
were synthesized for the expression in E. coli and yeast. The Odinarchaeota LCB4 ORFs (accession codes provided in Supplementary Table 2) were PCR amplified form MDA amplified environmental DNA isolated from the Lower Culex Basin Yellowstone National Park, USA as described ${ }^{94}$ and cloned into either pET28a (Vps23 and Vps28) or pET30 (Vps22 and Vps25) (Novagen), respectively. Amplified genes were cloned into the plasmids using the restriction sites placing the ORFs in frame with the plasmid-encoded hexa-histidine tags.

\section{Plasmids used in this study}

The Asgard genes obtained by gene synthesis were cloned into yeast two-hybrid $(\mathrm{Y} 2 \mathrm{H})$ vectors and $E$. coli expression vector. The plasmids for $\mathrm{Y} 2 \mathrm{H}$ are listed in Table S3. The E. coli expression plasmids are listed Table S6.

\section{Systematic, Reciprocal Yeast Two-Hybrid assays}

$\mathrm{Y} 2 \mathrm{H}$ assays were performed using the set of genes listed in Table S3. The plasmids used in this study are listed in Table S3. Indicated genes of interest were cloned both in "bait-ProteinA" and "prey-ProteinB" vectors or vice versa, which have DNA binding protein LexA and/or activation domain of Gal4p were cloned into pMM5 and pMM6 plasmids respectively ${ }^{95,96}$. Plasmids carrying these constructs were transformed into the yeast strains SGY37 (MATa) and YPH500 (MAT $\alpha$ ). Transformants with plasmids plexADBD (pMM5) and pGal4AD (pMM6) were selected on plates lacking Histidine or Leucine, respectively. After mating, the two strains carrying the desired plasmids were grown on YPD plates for 2 days at $30^{\circ} \mathrm{C}$ and replica plated on selection plates (without Histidine and Leucine) for 2 days at $30^{\circ} \mathrm{C}$ before the overlay. The interaction between the protein products fused to the DNA binding and activation domains were analyzed by the activity of $\beta$-galactosidase by the cleavage of X-Gal (BIO-37035, Bioline, UK). For detecting the $\beta$-galactosidase activity overlaying of low melting agarose with X-Gal (over lay mix was prepared freshly), overlay solution was added slowly on to the plates. Interaction of LexAProtein-A with Gal4-Protein-B resulted in the activation of expression of the lac $Z$ gene coding for $\beta$ galactosidase, converting $X-G a l$ to produce blue colour. Plates were monitored every 30 minutes to see the appearance of blue colour. Plates were scanned after $16 \mathrm{hr}$ of incubation with the X-Gal overlay mixture.

\section{Phylogenetic reconstruction \\ [A] UEV and E2}

Amino acid sequences of UEV domain-containing proteins, TSG101/Nps23 and UBC domain-containing proteins in $H$. sapiens, S. cerevisiae, D. discoideum, E. histolica, A. thaliana, C. marolae, T. brucei, $T$. pseudonana and T. parva were obtained from Uniprot. Asgard E2L proteins from Odinarchaeota (strain LCB_4), Heimdallarchaeota (strains AB125, LC2 and LC3), and Lokiarchaeota (strains GC14_75 and CR_4) were also obtained from Uniprot. These sequences were aligned with Mafft-linsi v7.450, and the resulting multiple-sequence alignment was used as query for a Psiblast (v2.10.0+) against all Asgard archaeal genomes (see Genome survey of protein homologs). All hits with e-values lower than 1e-5 were used together with query sequences and aligned using Mafft-linsi. The resulting alignment was trimmed using trimAl v1.4.rev22, and sequences containing over $60 \%$ gaps in the trimmed alignment were removed. The obtained alignment was used for a phylogenetic reconstruction with IQ-Tree 2.0$\mathrm{rc}^{89}$, under the model Q.pfam $+\mathrm{C} 20+\mathrm{G} 4+\mathrm{F}$, chosen by ModelFinder ${ }^{7}$ between combinations of empirical matrices (LG, WAG, JTT, and Q.pfam) with mixture models (C20, C40, and C60) and various rate heterogeneity (none, G4 and R4) and frequency (none, and F) and using 1000 ultrafast bootstrap pseudoreplicates. The resulting phylogeny was used as guide to reconstruct another tree under the PMSF approximation of the chosen model and using 100 non-parametric bootstrap pseudoreplicates. The resulting bootstrap trees were used both using the standard Felsenstein Bootstrap Proportion and the more recent Transfer Bootstrap Expectation ${ }^{98}$ interpretations.

[B] Vps22 and Vps36

Eukaryotic Vps22, Vps36 and Vps25 and Asgard Vps22/36 and Vps25 homologs sequences were downloaded from NCBI (Supplementary Data X). These 187 sequences were aligned using Mafft-linsi 
459 v7.450 and trimmed with trimAl with the parameter "-gappyout". A maximum-likelihood tree was then 460 reconstructed using IQ-Tree v2.0-rc1 under the model LG+C60+R4+F, using 1000 ultrafast bootstrap and $\mathrm{SH}$-approximate likelihood ratio test pseudoreplicates. In parallel, potential outgroup sequences (eukaryotic Rpc35/Rpc6, Asgard archaeal UFM1 and bacterial ScpB; Supplementary Data X) were downloaded and added to the previous sequences. Three additional Asgard archaeal sequences were found to contain potential plekstrin domains and were used as query for a Blast-p search against the Asgard proteomes to recruit homologs identified as hits with e-values lower than $1 \mathrm{e}-10$. The resulting set of 314 sequences was then aligned with Mafft-linsi v7.450 and trimmed with trimAl to remove all sites with over $90 \%$ gaps. The resulting trimmed alignment was used to reconstruct a maximumlikelihood tree using IQ-Tree v2.0-rc2 under the PMSF approximation ${ }^{99}$ of the LG+C60+R4+F model using 100 non-parametric bootstrap pseudoreplicates. The resulting bootstrap trees were used both using the standard Felsenstein Bootstrap Proportion and the more recent Transfer Bootstrap Expectation interpretations. To ensure we did not miss possible homologs of ESCRT-II sequences outside of Asgards, we used the previous set of $187 \mathrm{Vps} 22 / \mathrm{Vps} 36 / \mathrm{Vps} 25$ sequences as query for a psiblast search against the NR database (1 iteration, e-value threshold of 1e-10), and parsed the resulting 4745 hits to remove proteins originating from Asgard archaeal or eukaryotic genomes. After parsing, only 9 sequences remained, belonging to various putative archaea and bacteria. A Blast-p search of these sequences against NR confirmed that their best hits were Asgard archaea or eukaryotic sequences. We added these sequences to the previous $227 \mathrm{Vps} 22 / \mathrm{Vps} 36 / \mathrm{Vps} 25 /$ Outgroup sequences, aligned them with Mafft-linsi v7.450 and trimmed with trimAl to remove sites with over $50 \%$ gaps. We used this alignment to reconstruct a tree with IQ-Tree under the LG+C20+G4+F model, using 1000 ultrafast bootstrap and $\mathrm{SH}$-approximate likelihood ratio test pseudoreplicates. The resulting tree confirmed that these 9 homologs were well embedded in the clades of Asgard archaeal or eukaryotic Vps22, thus likely representing Asgard archaeal or eukaryotic Vps22 sequences that have been misclassified in public databases.

\section{bdSUMO tag vector and bdSUMO protease used in this study}

The vector carrying a 6-His residues (His-tag) followed by SUMO protein from Brachypodium distachyon were generated as described before ${ }^{100,101}$ with slight modification. The gene of $B$. distachyon SUMO protein (bdSUMO) was synthesized (IDT gBlock) and cloned into pET28a in frame with sequence encoding the N-terminal His-tag. The codon of the bdSUMO was optimized for the expression in E. coli K12 strain. The resulting vector, pSUMO was used to clone Heimdall ESCRT genes for their expression in E. coli BL21(DE3). To express and purify SUMO protease in B. distachyo, we synthesized genes of $B$. distachyo SENP1 whose codone were optimized for expression in $E$. coli (IDT gBlock). The gene fragment was cloned into pET28a vector in-frame with N-terminal His-tag. The protein, His-bdSENP1 was expressed in BL21(DE3) and purified and used for SUMO-TAG cleavage.

\section{Buffers used for protein purification}

The composition of ESCRT lysis buffer was as follows: $50 \mathrm{mM}$ Tris- $\mathrm{HCl}(\mathrm{pH} 7.5), 2.5 \mathrm{mM} \mathrm{MgCl} 2,150$ $\mathrm{mM} \mathrm{NaCl}, 2 \mathrm{mM}$ DTT, $2 \mathrm{mM}$ ATP, and $15 \mathrm{mM}$ Imidazole. The composition of ESCRT buffer was as follows: $50 \mathrm{mM}$ Tris-HCl (pH 7.5), $2.5 \mathrm{mM} \mathrm{MgCl} 2$ and $150 \mathrm{mM} \mathrm{NaCl}$. The composition of XL buffer is as follows; 20 mM HEPES-NaOH (pH 7.5), $150 \mathrm{mM} \mathrm{NaCl}$.

\section{Protein purification}

\section{(A) Heimdall proteins}

Purification of untagged proteins

The proteins were expressed as N-terminal His-SUMO fusions (His-SUMO) from a pSUMO vector. After the affinity purification using His-Nickel interaction, the His-SUMO was cleaved by His-bdSENP1 and both the N-terminal His-SUMO tag and His-bdSENP1 were absorbed on a Ni-NTA column. The untagged recombinant protein was further purified by size-exclusion chromatography (SEC). 
512

513

514

515

516

517

518

519

520

521

522

523

524

525

526

527

528

529

530

531

532

533

534

535

536

537

538

539

540

541

542

543

544

545

546

547

548

549

550

551

552

553

554

555

556

557

558

559

560

561

562

563

564

\section{Purification of ESCRT proteins}

[a] Heimdall Vps22 and Full-length or UEV-domain of Heimdall UEV-Vps28 (HeimAB125_14070) The $\mathrm{E}$. coli cells expressing these fusion proteins were lysed using a pressure homogenizer (Stanstead \#FPG12800, the 20-30 psi for several times precooled at $5^{\circ} \mathrm{C}$ ) instead of sonicator. Elution after the SEC was concentrated followed by high-speed centrifugation at $4^{\circ} \mathrm{C}(21000 \mathrm{xg}, 15 \mathrm{~min})$ to get rid of aggregation. The samples were snap-frozen and stored at $-80^{\circ} \mathrm{C}$.

[b] Ubiquitin with N-terminal His tag

Cells were re-suspended in lysis buffer containing $2 x$ concentration of PIC (Roche complete, EDTA-free \#05056489001) and $2 \mathrm{mM}$ PMSF. Cells were lysed using a pressure homogenizer (Stanstead \#FPG12800, the 20-30 psi for several times) precooled to $5^{\circ} \mathrm{C}$. After cell lysis, the total volume of the sample was $\sim 50 \mathrm{~mL}$. The insoluble fraction was removed by high-speed centrifugation at $4^{\circ} \mathrm{C}(25658 \mathrm{x}$ $\mathrm{g}$ for 1 hour, Thermo Fisher SCIENTIFIC \#A23-6 x 100 rotor). The supernatant was incubated with $2 \mathrm{ml}$ Ni-NTA resin (Thermo SCIENTIFIC \#88222) for 1 hour at $4^{\circ} \mathrm{C}$. The resin was washed with $200 \mathrm{~mL}$ icecold lysis buffer, followed by $150 \mathrm{ml}$ ESCRT-buffer. The bound protein was eluted with ESCRT-buffer containing $300 \mathrm{mM}$ imidazole. Elution fractions were combined and concentrated to a volume of $500 \mu \mathrm{l}$. The sample was spun at $21000 \mathrm{xg}$ for $15 \mathrm{~min}$ at $4^{\circ} \mathrm{C}$ and the supernatant was applied to $16 / 60$ sephacryl S-100 HR column equilibrated with ESCRT-buffer. The eluate was concentrated and spun at $4^{\circ} \mathrm{C}$ (21000xg, $15 \mathrm{~min}$ ) to get rid of aggregates. The samples were snap-frozen and stored at $-80^{\circ} \mathrm{C}$.

\section{(B) Odinarchaeal proteins}

Thermophilic Odinarchaeota proteins were expressed in Rosetta (DE3) pLysS Escherichia coli cells (Novagen). Cultures were grown at $37^{\circ} \mathrm{C}$ to an $\mathrm{OD}_{600}$ of 0.3 then cooled to $25^{\circ} \mathrm{C}$ and further grown to an $\mathrm{OD}_{600}$ of 0.6 and induced overnight with $0.33 \mathrm{mM}$ IPTG. Cells expressing the recombinant Odinarchaeota proteins were harvested by centrifugation, resuspended in $20 \mathrm{mM}$ Tris- $\mathrm{HCl}(\mathrm{pH} 8.0)$, $300 \mathrm{mM} \mathrm{NaCl}, 5 \%$ glycerol, $0.05 \%$ $\beta$-mercaptoethanol. 1X EDTA-free protease inhibitors (Complete cocktail, Roche) were added and cells were lysed by sonication and heat clarified at $60^{\circ} \mathrm{C}$ for $20 \mathrm{~min}$ before centrifugation at (14,000 r.p.m. for $10 \mathrm{~min})$ to remove insoluble material. Supernatants were filtered and then purified by IMAC by gravity flow to a column of Ni-NTA agarose (Qiagen). The columns were washed with resuspension buffer and then resuspension buffer plus $15 \mathrm{mM}$ imidazole. Proteins were then eluted in resuspension buffer plus $500 \mathrm{mM}$ imidazole. Fractions containing the purified proteins were pooled and concentrated before running a size-exclusion chromatography (SEC) step over a Superdex 200 16/600 column (GE Healthcare), in $20 \mathrm{mM}$ Tris- $\mathrm{HCl} \mathrm{pH} \mathrm{8,} 300 \mathrm{mM} \mathrm{NaCl}, 5 \%$ glycerol, $0.5 \mathrm{mM}$ dithiothreitol. N-terminal His-tags were then removed from the Odinarchaeota Vps23(TSG101) and Vps28 proteins by thrombin cleavage and further purification by SEC. Fractions containing the purified proteins were pooled, concentrated, aliquoted and flash frozen in liquid $\mathrm{N}_{2}$. Protein concentrations were quantified by UV spectrophotometry.

\section{Analytics Size Exclusion Chromatography}

Heimdallarchaeota Vps22 (27.9 kDa) was subjected to analytical SEC using a Superdex 200 16/600 size exclusion column (GE Healthcare). The sample was loaded onto the column in a buffer comprised of $20 \mathrm{mM}$ Tris $-\mathrm{HCl} \mathrm{pH} \mathrm{8.0,200} \mathrm{mM} \mathrm{NaCl}$ and $5 \%(\mathrm{v} / \mathrm{v})$ glycerol at a flow rate of $0.5 \mathrm{~mL} / \mathrm{min}$. The calibration curve was established under the same conditions using the following standard proteins (Sigma MWGF1000): carbonic anhydrase (CAN; $29 \mathrm{kDa}$ ), bovine serum albumin (BSA; $66 \mathrm{kDa})$, alcohol dehydrogenase (ADH; $150 \mathrm{kDa})$, beta-amylase (BAM; $200 \mathrm{kDa})$, apoferritin (AFE; $443 \mathrm{kDa})$ and thyroglobulin (TGL; $669 \mathrm{kDa}$ ).

Physical interaction between the Odinarchaeota ESCRT-I complex proteins (Vps23, Vps28 and ubiquitin) was examined by size-exclusion chromatography using an analytical Superdex S200 HR $10 / 300$ column (GE Healthcare). Prior to the gel filtration analyses, ESCRT-I complexes were formed at $60^{\circ} \mathrm{C}$ by mixing $250 \mu \mathrm{g}$ of each protein in a final volume of $500 \mu \mathrm{l}$ gel filtration buffer $(20 \mathrm{mM}$ Tris $[\mathrm{pH}$ 
602

603

604

605

606

607

608

609

610

611

612

$8.0], 150 \mathrm{mM} \mathrm{NaCl}, 5 \%$ glycerol, $1 \mathrm{mM}$ DTT). The complexes were subsequently spun at $16,000 \mathrm{~g}$ in a benchtop centrifuge for $5 \mathrm{~min}$ to remove any precipitated material, before loading onto the size exclusion chromatography column. $0.5 \mathrm{ml}$ fractions were collected and resolved by SDS-PAGE, on $15 \%$ polyacrylamide gels. The proteins were then visualized with Coomassie stain.

\section{Size exclusion chromatography-multi-angle laser light scattering (SEC-MALS)}

The molecular mass and oligomeric state of Heimdall Vps22 was determined in solution using SECMALS. Data were obtained with a Wyatt Heleosll18 angle light scattering machine connected to a Wyatt Optilab rEX online refractive index detector (Wyatt Technology). Samples were purified using a Superdex 200 increase 10/300 analytical gel filtration column (Cytiva) coupled to an Agilent 1200 series LC system at $0.5 \mathrm{ml} / \mathrm{min}$ in $20 \mathrm{mM}$ Tris-HCl pH 8.0, $200 \mathrm{mM} \mathrm{NaCl}$ buffer before detecting the light scattering and refractive index in a standard SEC-MALS format. Protein concentration was obtained from the excess differential refractive index of $0.185 \Delta R$ I for $1 \mathrm{~g} / \mathrm{ml}$ or using the sequence UV extinction coefficient of 0.964 at $280 \mathrm{~nm}$ for $1 \mathrm{mg} / \mathrm{ml}$ calculated by ProtParam. The determined protein concentration and scattering intensities were used to estimate the molecular mass from the intercept of a Debye plot using Zimm's model and the Wyatt ASTRA software. The experimental configuration was checked with a BSA standard, run in the same buffer and using the same sample injection volume of 100uL. The BSA monomer peak was utilised to examine the mass determination and to inspect the inter-detector delay volumes and band broadening parameters that were used during analysis in Wyatt's ASTRA software. The SEC chromatogram, showing RI as concentration signal, is shown in Figure 3B as blue and red lines for loaded sample concentration of 2 and $0.5 \mathrm{mg} / \mathrm{ml}$, respectively. The mass evaluated averaged over the central $75 \%$ of peak area is 54.4 and $54.2 \mathrm{kDa}$ for the two loadings indicating stable formation of dimer at SEC concentrations. The mass evaluated using UV as concentration source was $55.7 \mathrm{kDa}$ for the $2 \mathrm{mg} / \mathrm{ml}$ sample. The Odinarchaeota samples were analysed by SEC-MALS $(100 \mu$ l protein complex at $2 \mathrm{mg} / \mathrm{ml})$ were passed over a Superdex 200 10/300 Increase GL column (GE Healthcare), in $20 \mathrm{mM}$ Tris ( $\mathrm{pH} 8.0$ ), $300 \mathrm{mM} \mathrm{NaCl}$. The column output was fed into a miniDAWN TREOS MALS detector system with a $60 \mathrm{~mW}$ laser source at $664 \mathrm{~nm}$, and three fixed angle detectors at 49. 90, and 131 degrees (Wyatt Technology), followed by a Shimadzu RID-20A Refractive Index Detector at $30.5^{\circ} \mathrm{C}$.

\section{Circular Dichroism (CD)}

Proteins were buffer exchanged into freshly prepared buffer $(10 \mathrm{mM}$ potassium phosphate, $50 \mathrm{mM}$ sodium sulphate, $\mathrm{pH}$ 7.2) using PD-10 desalting prepacked columns (Sephadex G-25M, GE Healthcare) following manufactures instructions.

From the protein samples $(5 \mu \mathrm{M}) \mathrm{CD}$ spectra (in triplicate) were acquired using a Chirascan Plus Benchtop CD spectrophotometer over 180-260 nm with a bandwidth of $2 \mathrm{~nm}$ and a pathlength of $0.2 \mathrm{~mm}$. The mean buffer subtracted CD spectra (measured ellipticity: mdeg) were interpolated between 190-250 nm using Origin Pro 2018b and fitted to the BeStSel algorithm to determine the secondary structural elements ${ }^{102}$. Structural models for each ESCRT protein were generated using I-TASSER ${ }^{70-72}$ (or for full-length Vps25, trRosetta $^{79}$ ) after which the STRIDE web server ${ }^{103}$ was used to estimate secondary structure elements for comparison with the $C D$ derived estimations.

\section{$\Delta \mathrm{N} \_$Vps25 Crystallisation conditions}

An $\mathrm{N}$-terminally truncated $\mathrm{Vps} 25$ expression construct (removing the first 58 amino acids) was generated using the primers and Od $\Delta$ N_ESCIIV25forXhol and OdESCIIV25revXhol as described in Supplementary Table 2. The protein was purified as described above, except $5.25 \mathrm{mM}$ TCEP was used as the reducing agent in the final size exclusion chromatography step.

$\Delta \mathrm{N} \_$Vps25 crystals were grown by sitting-drop vapour diffusion using our in-house high-throughput crystallisation platform ${ }^{104}$. Vps 25 was used at a concentration of $21.4 \mathrm{mg} / \mathrm{ml}$ and the best crystals were obtained in the condition E12 of the Morpheus screen ${ }^{105}$ : [120 mM ethylene glycols, $100 \mathrm{mM}$ buffer 3 (26.7 $\mathrm{ml} 1 \mathrm{M}$ bicine plus $23.3 \mathrm{ml} 1 \mathrm{M}$ Trizma base), $12.5 \%$ (w/v) PEG 3350, $12.5 \%$ (w/v), $12.5 \%$ (w/v) 
617 PEG $1 \mathrm{~K}, 12.5 \%(\mathrm{w} / \mathrm{v}) \mathrm{MPD}$, $\mathrm{pH} 8.5$ at $20^{\circ} \mathrm{C}$ with a protein : reservoir ratio of $1: 4$ and a total volume of $6180.4 \mu \mathrm{l}$. The condition was already cryo-protected. Crystals were harvested by flash cooling in liquid 619 nitrogen.

\section{X-ray diffraction data collection}

Native diffraction data were collected at Diamond Light Source (Harwell, UK) at beamline I03. Data were collected over $360^{\circ}$ with $0.1^{\circ}$ oscillation (Supplemental Table 1), integrated with DIALS (https://doi.org/10.1107/S2059798317017235) and scaled/merged with Aimless

(https://www.ncbi.nlm.nih.gov/pmc/articles/PMC3689523/) from the CCP4 suite ${ }^{106}$. The crystals belong to the space group $P 2{ }_{1} 2{ }_{2}$, with unit cell dimensions of $a=101.23 \AA, b=31.5 \AA, c=59.5 \AA$ and one molecule per asymmetric unit. The crystals diffracted up to $1.8 \AA$. BALBES was used to determine initial phases by Molecular Replacement against the entire PDB ${ }^{107}$. Manual building was done in COOT $^{108}$ and refinement with REFMAC5. MOLPROBITY was used for model validation ${ }^{109}$. Statistics are listed in Table 1. The coordinates and structure factors of the Odinarchaeota Vps $25 \Delta \mathrm{N}$ crystal structure were deposited in the Protein Data Bank under accession code 7PB9.

\section{Chemical cross-linking of proteins}

[A] Vps22 dimer

Vps22 was diluted to $15 \mu \mathrm{M}$ after the buffer exchange to XL-buffer and incubated with $16 \mathrm{mM}$ EDC (Thermo Scientific, \#22980) and 16 mM Sulfo-NHS (Thermo Fisher Scientific, \#A39269) or 2 mM BS3 [bis(sulfosuccinimidyl)suberate, Creativemolecules, \#001SS] on ice for 1 or 2 hours, respectively. 55.6 $\mathrm{mM}$ Tris- $\mathrm{HCl}(\mathrm{pH} 6.8)$ was added into the mixture to quench the cross-linking reaction. The sample was incubated on ice for 10 min to quench the cross-linking reactions. The samples were loaded in SDSPAGE gels to separate individual or cross-linked proteins.

[B] Ubiquitin and UEV

The full length $(0.4 \mu \mathrm{M})$ or $1: 130$ amino acid residues containing UEV domain $(5 \mu \mathrm{M})$ of Heimdall_14070 were incubated with $0,1,2,4,8,16$ and $24 \mu \mathrm{M}$ Heimdall_14240 (wild-type or 145V) and 5 mM BS3 (Creativemolecules, \#001SS) on ice for 2 hours. The cross-linking reaction was quenched by addition of $50 \mathrm{mM}$ Tris- $\mathrm{HCl}$ ( $\mathrm{pH} \mathrm{6.8)}$. The samples were loaded in SDS-PAGE gels to separate individual or cross-linked proteins. 50 mM Ammonium Bicarbonate (\#A6141, Sigma) was used to dilute the sample using equal volume and reduced/alkylated using 10mM Tris-|(2-carboxyethyl) phosphine hydrochloride (TCEP) (\#148415000, ACROS Organics)/40 mM 2-chromoacetamide (CAA) (\#C4706-2G, Sigma) for 5 minutes at $70^{\circ} \mathrm{C}$. The samples were digested overnight at $37^{\circ} \mathrm{C}$ with $1 \mu \mathrm{g}$ trypsin (sequencing grade; \#V5111, Promega) per $100 \mu g$ of protein.

\section{Chemical cross-linking coupled with mass spectrometric analysis}

LC-MS was performed using Ultimate ${ }^{\circledR} 3000$ HPLC series for peptide concentration and separation. Nano Series TM Standards Columns were then utilised to separate the samples. A linear gradient from $4 \%$ to $25 \%$ solvent B (0.1\% formic acid in acetonitrile) was applied over 30 min, followed by $25 \%$ to $90 \%$ solvent B for $20 \mathrm{~min}$. Peptides were eluted using at a rate of $250 \mathrm{~nL} \mathrm{~min}{ }^{-1}$ using a Triversa Nanomate nano spray into the Orbitrap Fusion mass spectrometer (ThermoScientific). Mass scan range of 3751500 were used for the peptide precursors at $120 \mathrm{~K}$ resolution, with automatic gain control of $4 \times 10^{5}$. Precursor ions range of 2-7 were isolated and fragmented using Higher-energy Collisional Dissociation (HCD) fragmentation using the Orbitrap detector at a resolution of $30 \mathrm{~K}$. MS/MS fragmentation was performed using a collision energy of 33\%, with a maximum injection time of $200 \mathrm{~ms}$ and automatic gain control of $1 \times 10^{4}$. Dynamic exclusion duration of $45 \mathrm{~s}$ with $5 \mathrm{ppm}$ tolerance was used for the selected precursor and its isotopes. The instrument was run with a cycle time of $2 \mathrm{~s}$. $20 \mathrm{ul}$ of the samples were injected into the nano LC-ESI-MS/MS using an Ultimate 3000/Orbitrap Fusion (Thermo Scientific) using a 60 -minute LC separation over a $50 \mathrm{~cm}$ column. The ProteoWizard MSConvert toolkit ${ }^{42}$ was used to convert the raw data files into .mgf format. Scaffold Proteome Software was used for sequence visualization and coverage. Cross-linked peptides were analysed using the Stavrox software ${ }^{110}$, using the in-built parameters for either BS3 or EDC. Precursor and fragment ion tolerance were set to $10 \mathrm{ppm}$. 
The spectra were manually inspected, and continuous fragment ions were expected to be seen for both peptides. Cross-linked peptides were identified in two replicate datasets. Detected peptides were listed in Table S4.

\section{ACKNOWLEDGEMENTS}

We thank Dr. Masayuki Onishi (Duke University) for critical reading and constructive comments on a early version of the manuscript. Computational analysis was facilitated by resources provided by the Swedish National Infrastructure for Computing (SNIC) at the Uppsala Multidisciplinary Center for Advanced Computational Science (UPPMAX), partially funded by the Swedish Research Council through grant agreement no. 2018-05973. We thank the Warwick Proteomics RTP for mass spectrometry. MKB was supported by the Wellcome Trust (WT101885MA) and the European Research Council (ERC-2014-ADG No. 671083). Work by the NR laboratory was supported by start-up funds from the Division of Biomedical and Life Sciences (BLS, Lancaster University) and a Leverhulme Research Project Grant (RPG-2019-297). NR would like to thank Johanna Syrjanen for performing trial expressions of the Odinarchaeota ESCRT proteins, and Joseph Maman for helpful discussion regarding the SEC-MALS. NR, WX and AP would like to thank Charley Lai and Siu-Kei Yau for assistance with initial Odinarchaeota ESCRT protein purifications. DPS and BB would like to thank Chris Johnson at the MRC LMB Biophysics facility for performing the SEC-MALS assay on Heimdall Vps22. TH, HH, MB, RS, JL, D Tamarit, TE, DPS and BB received support from a Wellcome Trust collaborative award (203276/Z/16/Z). BB and DPS were supported by the MRC. DT was supported by the Swedish Research Council (International Postdoc grant 2018-06609).

\section{AUTHOR CONTRIBUTIONS}

$\mathrm{TH}, \mathrm{BB}, \mathrm{NR}, \mathrm{MB}$ conceived and integrated the overall study.

TH, SP, NR, DPS, D Tamarit, RS made all figures.

$\mathrm{TH}, \mathrm{MB}, \mathrm{NR}, \mathrm{DPS}, \mathrm{D}$ Tamarit, and BB wrote the first draft of the manuscript

All authors contributed to reading and revising the manuscript.

TH (under supervision of MB): carried out all Heimdall experiments in Figures 2 and 3 (except for SECMALS: DPS- and the analysis of mass spectrometry-HH), all iTASSER modelling of Heimdall proteins, and helped make $\mathrm{Y} 2 \mathrm{H}$ plasmids, and carried out preliminary phylogenetic analysis.

$\mathrm{SP}$ (under supervision of $\mathrm{MB}$ ): made most plasmids for $\mathrm{Y} 2 \mathrm{H}$ and carried out the entire pairwise $\mathrm{Y} 2 \mathrm{H}$ screen.

DP (under supervision of NR): Expressed and purified the Odinarchaeota ESCRT proteins (with AH, WX, AP, MM and NR), performed the CD analyses (with MM and DT), the Odin SEC-MALS analyses (with NR and DR) performed the ESCRT-II molecular modelling (with NR) and designed and purified Vps25 constructs for crystallography (with NR).

DPS (under supervision of BB): Expressed, purified, and carried out the analytical gel filtration on Heimdall Vps22, collected sequences for phylogenetic studies and helped in the bioinformatics analyses of $D$ Tamarit.

RS (under supervision of $\mathrm{JL}$ ): solved the structure of $\mathrm{Vps} 25$

D Tamarit (under supervision of TE): performed all genomic and phylogenetic analyses.

HMAH: Processed and analyzed XL-MS data.

SP, DP, DPS, RS and D Tamarit contributed equally to this work and are listed alphabetically.

\section{FIGURE LEGENDS}

Figure 1. The ASGARD archaea, Lokiarchaeota, Heimdallarchaeota, Helarchaeota, and Odinachaeota, have genes that comprise the ubiquitin-ESCRT pathway.

(A) List of proteins in the Asgard archaea and eukaryotic Ubiquitin-ESCRT (Ub-ESCRT) pathway. 
(B) Co-location of Ub/ESCRT protein-encoding genes in Heimdall- (i; 22 genomes), Hel- (ii; 9 genomes), Loki- (iii; 29 genomes) and Thorarchaeota (iv; 30 genomes). A colour gradient indicates the fraction of genomes in which a pair of genes was found to co-locate at less than $10 \mathrm{~kb}$, and white cells indicate gene pairs found to never co-exist in any genome of the same phylum.

(C) Synteny plot of selected genomes. Arrows represent genes and are coloured if their products were annotated as containing diagnostic domains for Ub/ESCRT proteins (see Methods). Genes encoding Vps23/37 in the vicinity of the ESCRT gene cluster in Helarchaeote Hel_GB_B, Heimdallarchaeote B3_Heim and Odinarchaeote LCB_4 were only found to contain E2 dōmains, but were deemed $\mathrm{Vps} 23 / 37$ through alignment (Suppl. Fig. X). A gene encoding a fusion of $\mathrm{Vps} 23 / 37$ and $\mathrm{Vps} 28$ is colored as both orange and red. Genome regions are plotted at $2 \mathrm{~kb}$ of ubiquitin or ESCRT protein-encoding genes (coloured), or until a contig boundary (thicker vertical lines). Similarity lines indicate bestreciprocal BLAST-p (Altschul et al $1990 \mathrm{~J}$ Mol Biol 215: 403-410) hits with an e-value lower than 1e-5. The names of the organisms used for experimental analyses in later sections are marked in orange.

(D) Phylogenetic reconstruction of Vps22 and Vps36. Unrooted maximum likelihood phylogenetic tree of Vps22 (blue), Vps36 (purple) and Vps25 (orange) and outgroup (black) sequences. The tree was reconstructed using IQ-Tree under the LG+C60+R4+F model. Support values are only shown for the deeper branches connecting gene homologs and represent standard Felsenstein bootstrap proportions (upper left) or transfer-bootstrap expectation (TBE) (lower right) values based on 100 bootstrap pseudoreplicates. The full tree with all leaf and support labels is shown in Suppl. Figure X.

(E) Phylogenetic reconstruction of UEV domain-containing proteins and E2 ubiquitin-conjugating enzymes. Unrooted maximum likelihood phylogenetic tree of the UEV domain-containing proteins (orange, red) and E2 ubiquitin-conjugating proteins (gray, black) in Eukarya and Asgard archaea. The tree was reconstructed using IQ-Tree under the Q.pfam+C20+G4+F+PMSF model. Support values are only shown for the deeper branches, following the same pattern as in (D).

\section{Figure 2. Assembly of Asgard ESCRT-I complexes with ubiquitin binding to the UEV domain of Vps23(TSG101).}

(A) Schematic diagram of the domain structure of HeimAB125 Vps28 and the truncation design used in the experiments. The N-terminal ubiquitin E2 variant domain ("UEV") and the core domain of Vps28 ("Vps28") identified previously are highlighted.

(B) A model of the three-dimensional structure of HeimAB125 UEV. The three-dimensional structure model was created by templating the structure of the budding yeast Vps23 bound to ubiquitin. Vps23 (PDB: 1UZXa, light green) and HeimAB125 UEV (model structure, purple) are superimposed. Ubiquitin bound to Vps23 (PDB: 1UZXb) is shown in yellow. Two ubiquitin-binding "arms" of Vps23, "beta-hairpin Tongue" and "Lip" are highlighted by circles with dashed lines.

(C) The three-dimensional structure model of HeimAB125 Ubiquitin was created and superimposed with the structure of Ubiquitin in complex with Vps23 (PDB: 1UZXb). Ubiquitin (PDB: 1UZXb, yellow), model structure of HeimAB125 Ubiquitin (light blue), Vps23 (PDB: 1UZXa, light green) were shown. An amino acid residue (Val45) on the model structure located in the Ubiquitin hydrophobic patch, which is important for Ubiquitin-UEV interactions, is highlighted in magenta. The structure of HeimAB125 ubiquitin is illustrated in the ribbon diagram (i) and the surface model (ii).

(D) HeimAB125 UEV binds ubiquitin in a manner dependent on a hydrophobic patch. The interaction between HeimAB125 Ubiquitin (wild-type or V45D mutant) and UEV-Vps28 (full-length, top panel) or UEV domain (bottom panel) was tested by BS3-mediated chemical cross-linking, followed by SDSPAGE to detect the changes in their molecular weight. 
817

818

819

820

821

822

823

824

825

826

827

828

(E) Size-exclusion chromatography analysis of the Odinarchaeota ESCRT-I subcomplex assembly. Physical interaction between the Odinarchaeota ESCRT-I subcomplex and ubiquitin as demonstrated by size exclusion chromatography. From Top to Bottom: Vps28 protein only (top); Vps23(TSG101) protein only; ubiquitin only; Vps28 pre-incubated with ubiquitin (no interaction); Vps23 (TSG101) preincubated with Vps28 (stable complex formation); Vps23 (TSG101) pre-incubated with Vps28 and ubiquitin (bottom - ubiquitin binds to the Vps23(TSG1010)/Vps28 complex, via the UEV domain of Vps23(TSG101). For additional controls see Supplementary Figure S10). All proteins were separated on a Superdex S200 HR 10/300 size exclusion chromatography column. The relative elution volumes of the size standards $\beta$-amylase (200 kDa), alcohol dehydrogenase (150 kDa), bovine serum albumin (BSA) (66 kDa) and carbonic anhydrase (29 kDa) and cytochrome-c (12.4 kDa) are also indicated (in grey). Eluted fractions were resolved by SDS-PAGE and visualised by Coomassie stain. Left: chromatography UV traces (at $280 \mathrm{~nm}$ ) for the respective elution profiles.

\section{Figure 3. Heimdall Vps22 forms stable dimers.}

(A) Elution chromatogram of Vps22 (27.9 kDa) using a Superdex 200 16/600 size exclusion column. The inset shows the column calibration curve established with standard proteins (see Methods section). Grey lines indicate the Ve/Vo and predicted molar mass ( $85 \mathrm{kDa}$ ) of Vps22. This assay suggests that this protein forms a trimer or an elongated dimer.

(B) SEC-MALS analysis of Heimdall Vps22 using a Superdex 200 increase 10/300 analytical column. The chromatograms display the calculated molar mass of the peaks $(\mathrm{kDa})$ and refractive indexes (A.U.) as dots and lines, respectively, for loaded sample concentrations of 2.0 (blue) and 0.5 (red) mg/ml. The estimated masses are 54.4 and $54.2 \mathrm{kDa}$ for the two protein concentrations, indicating stable formation of a Vps22 dimer, as the theoretical dimer mass is $55.9 \mathrm{kDa}$.

(C) Purified HeimAB125 Vps22 showed slower migration on SDS-PAGE gel by chemical crosslinking, whose mobility is consistent with that of a cross-linked dimer.

(D and E) A model structure of HeimAB125 Vps22 superimposed on Vps22 in the structure of ESCRTII complex (3CUQ). Side chains of dimerized peptide around 160:166 aa (ii) and 41-47 aa (ii) on the Vps22 model structure were shown in the panel. The cross-linked positions on the peptide sequence were highlighted in red.

\section{Figure 4. Crystal structure of the Odinarchaeota $\operatorname{Vps} 25 \Delta \mathrm{N}$ tandem winged helix (WH) domain.}

(A) Archaeal Vps $25 \Delta \mathrm{N}$ tandem $\mathrm{WH}$ domain structure coloured from blue to red (N-terminus to Cterminus) shown in ribbon form, with secondary structural sequence elements indicated.

(B) Structural alignment of the Odinarchaeota $\mathrm{Vps} 25 \Delta \mathrm{N}$ (red) with Vps25 from S. cerevisiae (green) (PDB: 1XB4) and $H$. sapiens (blue) (PDB: 2ZME).

(C) Superposition of the N- (blue) and C-terminal (pink) archaeal Vps25 WH domains. Refinement and model statistics are shown in Supplementary Table 1.

Figure 5. Systematic reciprocal Yeast two-hybrid assays between Asgard ESCRT proteins and new insight gained from investigating ESCRT from Asgard.

(A) Summary of $\mathrm{Y} 2 \mathrm{H}$ interactions. Molecules related to the Ub-ESCRT pathway found in Lokiarchaeota (Lokiarch), Heimdallarchaeota (HeimAB125), Thorarchaeota, and 
829

830

831

832

833

834

835

836

837

838

839

840

841

842

843

844

845

846

847

848

849

850

851

852

853

854

855

856

857

858

859

860

861

862

863

864

865

866

867

868

869

870

871

872

873

874

875

876

877

878

Odinachaeota (OdinLCB4) were examined comprehensively using $\mathrm{Y} 2 \mathrm{H}$, and the detected interactions are illustrated (see also Figure $\mathrm{S} 6$ for the individual results)

(B) Schematic representation of the arrangement of the Asgard ESCRT pathway based on this work. In the Heimdallarcheota and Lokiarchaeota ESCRT-II complexes the Vps22 subunit forms a homodimer comparible to the eukaryotic Vps22/Nps36 ESCRT-II heterodimeric stalk. In Odinarcheota, however, the Vps22 homologue does not appear to dimerize and as yet undetermined factor therefore likely bridges the interaction between the ESCRT-I and -II subcomplexes. The Odinarcheota $\mathrm{Vps} 23$ homologue forms a dimer thereby presenting two ubiquitin-binding UEV domains. The Vps23 dimer interacts with a single Vps28 protein thus forming a tripartite complex, reminiscent of the eukaryotic $\mathrm{Vps} 37 / \mathrm{Vps} 23 / \mathrm{Vps} 28$ complex. In Heimdallarcheota the Vps23 and Vps28 functions are fused in a single protein that also dimerises. Compare with the eukaryotic arrangement as shown in Supplementary Figure S1.

\section{References}

1 Henne, W. M., Buchkovich, N. J. \& Emr, S. D. in Developmental Cell Vol. 21 77-91 (Cell Press, 2011).

2 Piper, R. C., Cooper, A. A., Yang, H. \& Stevens, T. H. VPS27 controls vacuolar and endocytic traffic through a prevacuolar compartment in Saccharomyces cerevisiae. The Journal of cell biology 131, 603-617, doi:10.1083/jcb.131.3.603 (1995).

$3 \quad \mathrm{Li}$, Y., Kane, T., Tipper, C., Spatrick, P. \& Jenness, D. D. Yeast mutants affecting possible quality control of plasma membrane proteins. Molecular and cellular biology 19, 3588-3599, doi:10.1128/mcb.19.5.3588 (1999).

4 Rieder, S. E., Banta, L. M., Köhrer, K., McCaffery, J. M. \& Emr, S. D. Multilamellar endosomelike compartment accumulates in the yeast vps28 vacuolar protein sorting mutant. Molecular Biology of the Cell 7, 985-999, doi:10.1091/mbc.7.6.985 (1996).

5 Babst, M., Odorizzi, G., Estepa, E. J. \& Emr, S. D. Mammalian Tumor Susceptibility Gene 101 (TSG101) and the Yeast Homologue, Vps23p, Both Function in Late Endosomal Trafficking. Traffic 1, 248-258, doi:10.1034/j.1600-0854.2000.010307.x (2000).

6 Munn, A. L. \& Riezman, H. Endocytosis is required for the growth of vacuolar $\mathrm{H}(+)$-ATPasedefective yeast: identification of six new END genes. The Journal of Cell Biology 127, 373-386, doi:10.1083/jcb.127.2.373 (1994).

7 Nothwehr, S. F., Bryant, N. J. \& Stevens, T. H. The Newly Identified Yeast GRD Genes Are Required for Retention of Late-Golgi Membrane Proteins. Report No. 9740312292, 2700-2707 (1996).

8 Babst, M., Sato, T. K., Banta, L. M. \& Emr, S. D. Endosomal transport function in yeast requires a novel AAA-type ATPase, Vps4p. EMBO Journal 16, 1820-1831, doi:10.1093/emboj/16.8.1820 (1997).

9 Katzmann, D. J., Babst, M. \& Emr, S. D. Ubiquitin-dependent sorting into the multivesicular body pathway requires the function of a conserved endosomal protein sorting complex, ESCRT-I. Cell 106, 145-155, doi:10.1016/S0092-8674(01)00434-2 (2001).

10 Kranz, A., Kinner, A. \& Kölling, R. A Family of Small Coiled-Coil-forming Proteins Functioning at the Late Endosome in Yeast. Molecular Biology of the Cell 12, 711-723, doi:10.1091/mbc.12.3.711 (2001).

11 Babst, M., Katzmann, D. J., Snyder, W. B., Wendland, B. \& Emr, S. D. Endosome-associated complex, ESCRT-II, recruits transport machinery for protein sorting at the multivesicular body. Developmental cell 3, 283-289, doi:10.1016/S1534-5807(02)00219-8 (2002). 
87912 Katzmann, D. J., Stefan, C. J., Babst, M. \& Emr, S. D. Vps27 recruits ESCRT machinery to endosomes during MVB sorting. The Journal of Cell Biology 162, 413-423, doi:10.1083/JCB.200302136 (2003).

\section{Bilodeau, P. S., Winistorfer, S. C., Kearney, W. R., Robertson, A. D. \& Piper, R. C. Vps27-Hse1} and ESCRT-I complexes cooperate to increase efficiency of sorting ubiquitinated proteins at the endosome. J Cell Biol 163, 237-243, doi:10.1083/jcb.200305007 (2003).

14 Shih, S. C. et al. Epsins and Vps27p/Hrs contain ubiquitin-binding domains that function in receptor endocytosis. Nature Cell Biology 4, 389-393, doi:10.1038/ncb790 (2002).

15 Odorizzi, G., Babst, M. \& Emr, S. D. Fab1p Ptdlns(3)P 5-Kinase Function Essential for Protein Sorting in the Multivesicular Body. Cell 95, 847-858, doi:10.1016/S0092-8674(00)81707-9 (1998).

16 von Schwedler, U. K. et al. The protein network of HIV budding. Cell 114, 701-713, doi:10.1016/S0092-8674(03)00714-1 (2003).

17 Strack, B., Calistri, A., Craig, S., Popova, E. \& Göttlinger, H. G. AIP1/ALIX is a binding partner for HIV-1 p6 and EIAV p9 functioning in virus budding. Cell 114, 689-699, doi:10.1016/S00928674(03)00653-6 (2003).

18 Martin-Serrano, J., Yarovoy, A., Perez-Caballero, D., Bieniasz, P. D. \& Yaravoy, A. Divergent retroviral late-budding domains recruit vacuolar protein sorting factors by using alternative adaptor proteins. Proceedings of the National Academy of Sciences of the United States of America 100, 12414-12419, doi:10.1073/pnas.2133846100 (2003).

19 Carlton, J. G. \& Martin-Serrano, J. Parallels between cytokinesis and retroviral budding: a role for the ESCRT machinery. Science (New York, N.Y.) 316, 1908-1912, doi:10.1126/science.1143422 (2007).

20 Morita, E. et al. Human ESCRT and ALIX proteins interact with proteins of the midbody and function in cytokinesis. The EMBO Journal 26, 4215-4227, doi:10.1038/SJ.EMBOJ.7601850 (2007).

21 VerPlank, L. et al. Tsg101, a homologue of ubiquitin-conjugating (E2) enzymes, binds the L domain in HIV type 1 Pr55Gag. Proceedings of the National Academy of Sciences 98, 77247729, doi:10.1073/PNAS.131059198 (2001).

22 Martin-Serrano, J., Zang, T. \& Bieniasz, P. D. HIV-1 and Ebola virus encode small peptide motifs that recruit Tsg101 to sites of particle assembly to facilitate egress. Nature Medicine 7, 13131319, doi:10.1038/nm1201-1313 (2001).

23 Skowyra, M. L., Schlesinger, P. H., Naismith, T. V. \& Hanson, P. I. Triggered recruitment of ESCRT machinery promotes endolysosomal repair. Science (New York, N.Y.) 360, eaar5078eaar5078, doi:10.1126/science.aar5078 (2018).

24 Jimenez, A. J. et al. ESCRT machinery is required for plasma membrane repair. Science (New York, N.Y.) 343, 1247136-1247136, doi:10.1126/science.1247136 (2014).

25 Scheffer, L. L. et al. Mechanism of Ca2+-triggered ESCRT assembly and regulation of cell membrane repair. Nature Communications 5, 5646-5646, doi:10.1038/ncomms6646 (2014).

26 Choi, H. W. et al. Perivascular dendritic cells elicit anaphylaxis by relaying allergens to mast cells via microvesicles. Science (New York, N.Y.) 362, eaao0666-eaao0666, doi:10.1126/science.aao0666 (2018).

27 Vietri, M. et al. Spastin and ESCRT-III coordinate mitotic spindle disassembly and nuclear envelope sealing. Nature 522, 231-235, doi:10.1038/nature14408 (2015).

28 Zhang, H. et al. Endocytic Pathways Downregulate the L1-type Cell Adhesion Molecule Neuroglian to Promote Dendrite Pruning in Drosophila. Developmental Cell 30, 463-478, doi:10.1016/j.devcel.2014.06.014 (2014).

29 Loncle, N., Agromayor, M., Martin-Serrano, J. \& Williams, D. W. An ESCRT module is required for neuron pruning. Scientific Reports 5, 8461-8461, doi:10.1038/srep08461 (2015).

30 Matusek, T. et al. The ESCRT machinery regulates the secretion and long-range activity of Hedgehog. Nature 516, 99-103, doi:10.1038/nature13847 (2014).

31 Olmos, Y., Hodgson, L., Mantell, J., Verkade, P. \& Carlton, J. G. ESCRT-III controls nuclear envelope reformation. Nature 522, 236-239, doi:10.1038/nature14503 (2015). 
93232 Denais, C. M. et al. Nuclear envelope rupture and repair during cancer cell migration. Science

933

934

935

936

937

938

939

940

941

942

943

944

945

946

947

948

949

950

951

952

953

954

955

956

957

958

959

960

961

962

963

964

965

966

967

968

969

970

971

972

973

974

975

976

977

978

979

980

981

982

983
352, 353-358, doi:10.1126/science.aad7297 (2016).

33 Raab, M. et al. ESCRT III repairs nuclear envelope ruptures during cell migration to limit DNA damage and cell death. Science 352, 359-362, doi:10.1126/science.aad7611 (2016).

34 Frost, A. et al. Functional repurposing revealed by comparing S. pombe and S. cerevisiae genetic interactions. Cell 149, 1339-1352, doi:10.1016/j.cell.2012.04.028 (2012).

35 Webster, B. M., Colombi, P., Jäger, J. \& Patrick Lusk, C. Surveillance of nuclear pore complex assembly by ESCRT-III/Vps4. Cell 159, 388-401, doi:10.1016/j.cell.2014.09.012 (2014).

36 Zhou, F. et al. Rab5-dependent autophagosome closure by ESCRT. Journal of Cell Biology 218, 1908-1927, doi:10.1083/JCB.201811173 (2019).

37 Takahashi, Y. et al. An autophagy assay reveals the ESCRT-III component CHMP2A as a regulator of phagophore closure. Nature Communications 9, 1-13, doi:10.1038/s41467-01805254-w (2018).

38 Sahu, R. et al. Microautophagy of Cytosolic Proteins by Late Endosomes. Developmental Cell 20, 131-139, doi:10.1016/j.devcel.2010.12.003 (2011).

39 Radulovic, M. et al. <scp>ESCRT</scp> -mediated lysosome repair precedes lysophagy and promotes cell survival. The EMBO Journal 37, e99753-e99753, doi:10.15252/embj.201899753 (2018).

40 Raiborg, C. \& Stenmark, H. in Nature Vol. 458 445-452 (Nature Publishing Group, 2009).

41 Raiborg, C. et al. Hrs sorts ubiquitinated proteins into clathrin-coated microdomains of early endosomes. Nature Cell Biology 4, 394-398, doi:10.1038/ncb791 (2002).

42 Bilodeau, P. S., Urbanowski, J. L., Winistorfer, S. C. \& Piper, R. C. The Vps27p-Hse1p complex binds ubiquitin and mediates endosomal protein sorting. Nature Cell Biology 4, 534-539, doi:10.1038/ncb815 (2002).

43 Babst, M., Wendland, B., Estepa, E. J. \& Emr, S. D. The Vps4p AAA ATPase regulates membrane association of a $\mathrm{Vps}$ protein complex required for normal endosome function. EMBO Journal 17, 2982-2993, doi:10.1093/emboj/17.11.2982 (1998).

44 Babst, M., Katzmann, D. J., Estepa-Sabal, E. J., Meerloo, T. \& Emr, S. D. ESCRT-III: An endosome-associated heterooligomeric protein complex required for MVB sorting. Developmental Cell 3, 271-282, doi:10.1016/S1534-5807(02)00220-4 (2002).

45 Hanson, P. I., Roth, R., Lin, Y. \& Heuser, J. E. Plasma membrane deformation by circular arrays of ESCRT-III protein filaments. The Journal of Cell Biology 180, 389-402, doi:10.1083/JCB.200707031 (2008).

46 Teis, D., Saksena, S. \& Emr, S. D. Ordered Assembly of the ESCRT-III Complex on Endosomes Is Required to Sequester Cargo during MVB Formation. Developmental Cell 15, 578-589, doi:10.1016/J.DEVCEL.2008.08.013 (2008).

47 Lata, S. et al. Helical Structures of ESCRT-III Are Disassembled by VPS4. Science 321, 13541357, doi:10.1126/SCIENCE.1161070 (2008).

48 Chiaruttini, N. et al. Relaxation of Loaded ESCRT-III Spiral Springs Drives Membrane Deformation. Cell 163, 866-879, doi:10.1016/j.cell.2015.10.017 (2015).

49 Mierzwa, B. E. et al. Dynamic subunit turnover in ESCRT-III assemblies is regulated by Vps4 to mediate membrane remodelling during cytokinesis. Nature Cell Biology 19, 787-798, doi:10.1038/ncb3559 (2017).

50 Maity, S. et al. VPS4 triggers constriction and cleavage of ESCRT-III helical filaments. Science Advances 5, eaau7198-eaau7198, doi:10.1126/sciadv.aau7198 (2019).

51 Teis, D., Saksena, S., Judson, B. L. \& Emr, S. D. ESCRT-II coordinates the assembly of ESCRTIII filaments for cargo sorting and multivesicular body vesicle formation. The EMBO Journal 29, 871-883, doi:10.1038/emboj.2009.408 (2010).

52 Saksena, S., Wahlman, J., Teis, D., Johnson, A. E. \& Emr, S. D. Functional reconstitution of ESCRT-III assembly and disassembly. Cell 136, 97-109, doi:10.1016/j.cell.2008.11.013 (2009). 
$98454 \quad$ Leung, K. F., Dacks, J. B. \& Field, M. C. Evolution of the Multivesicular Body ESCRT Machinery; Retention Across the Eukaryotic Lineage. Traffic 9, 1698-1716, doi:10.1111/j.16000854.2008.00797.x (2008).

55 Obita, T. et al. Structural basis for selective recognition of ESCRT-III by the AAA ATPase Vps4. Nature 449, 735-739, doi:10.1038/nature06171 (2007).

56 Samson, R. Y., Obita, T., Freund, S. M., Williams, R. L. \& Bell, S. D. A role for the ESCRT system in cell division in archaea. Science 322, 1710-1713, doi:10.1126/science.1165322 (2008).

57 Spang, A. et al. Complex archaea that bridge the gap between prokaryotes and eukaryotes. Nature 521, 173-179, doi:10.1038/nature14447 (2015).

58 Zaremba-Niedzwiedzka, K. et al. Asgard archaea illuminate the origin of eukaryotic cellular complexity. Nature, doi:10.1038/nature21031 (2017).

59 Lindås, A.-C., Karlsson, E. A., Lindgren, M. T., Ettema, T. J. G. \& Bernander, R. A unique cell division machinery in the Archaea. Proceedings of the National Academy of Sciences of the United States of America 105, 18942-18946, doi:10.1073/pnas.0809467105 (2008).

60 Snyder, J. C., Samson, R. Y., Brumfield, S. K., Bell, S. D. \& Young, M. J. Functional interplay between a virus and the ESCRT machinery in Archaea. Proceedings of the National Academy of Sciences of the United States of America 110, 10783-10787, doi:10.1073/pnas.1301605110 (2013).

61 Gupta, T. K. et al. Structural basis for VIPP1 oligomerization and maintenance of thylakoid membrane integrity. Cell 184, 3643-3659 e3623, doi:10.1016/j.cell.2021.05.011 (2021).

62 Junglas, B. et al. PspA adopts an ESCRT-III-like fold and remodels bacterial membranes. Cell 184, 3674-3688 e3618, doi:10.1016/j.cell.2021.05.042 (2021).

63 Liu, J. et al. Bacterial Vipp1 and PspA are members of the ancient ESCRT-III membraneremodeling superfamily. Cell 184, 3660-3673 e3618, doi:10.1016/j.cell.2021.05.041 (2021).

64 Nunoura, T. et al. Insights into the evolution of Archaea and eukaryotic protein modifier systems revealed by the genome of a novel archaeal group. Nucleic Acids Research 39, 3204-3223, doi:10.1093/nar/gkq1228 (2011).

65 Hennell James, R. et al. Functional reconstruction of a eukaryotic-like E1/E2/(RING) E3 ubiquitylation cascade from an uncultured archaeon. Nature Communications 8, 1-15, doi:10.1038/s41467-017-01162-7 (2017).

66 Imachi, H. et al. Isolation of an archaeon at the prokaryote-eukaryote interface. Nature 577, 519525, doi:10.1038/s41586-019-1916-6 (2020).

67 Seitz, K. W. et al. Asgard archaea capable of anaerobic hydrocarbon cycling. Nature Communications 10, 1-11, doi:10.1038/s41467-019-09364-x (2019).

68 Gilchrist, C. L. M. \& Chooi, Y.-H. clinker \&amp; clustermap.js: automatic generation of gene cluster comparison figures. Bioinformatics, doi:10.1093/bioinformatics/btab007 (2021).

69 Sancho, E. et al. Role of UEV-1, an Inactive Variant of the E2 UbiquitinConjugating Enzymes, in In Vitro Differentiation and Cell Cycle Behavior of HT-29-M6 Intestinal Mucosecretory Cells. Molecular and Cellular Biology 18, 576-589, doi:10.1128/mcb.18.1.576 (1998).

70 Roy, A., Kucukural, A. \& Zhang, Y. I-TASSER: A unified platform for automated protein structure and function prediction. Nature Protocols 5, 725-738, doi:10.1038/nprot.2010.5 (2010).

71 Yang, J. et al. in Nature Methods Vol. 12 7-8 (Nature Publishing Group, 2014).

72 Zhang, Y. I-TASSER server for protein 3D structure prediction. BMC Bioinformatics 9, 40-40, doi:10.1186/1471-2105-9-40 (2008).

73 Kostelansky, M. S. et al. Molecular architecture and functional model of the complete yeast ESCRT-I heterotetramer. Cell 129, 485-498, doi:10.1016/j.cell.2007.03.016 (2007).

74 Teo, H. et al. ESCRT-I Core and ESCRT-II GLUE Domain Structures Reveal Role for GLUE in Linking to ESCRT-I and Membranes. Cell 125, 99-111, doi:10.1016/J.CELL.2006.01.047 (2006).

75 Boura, E. et al. Solution structure of the ESCRT-I and -II supercomplex: implications for membrane budding and scission. Structure (London, England : 1993) 20, 874-886, doi:10.1016/j.str.2012.03.008 (2012). 
1035

1036

1037

1038

1039

1040

1041

1042

1043

1044

1045

1046

1047

1048

1049

1050

1051

1052

1053

1054

1055

1056

1057

1058

1059

1060

1061

1062

1063

1064

1065

1066

1067

1068

1069

1070

1071

1072

1073

1074

1075

1076

1077

1078

1079

1080

1081

1082

1083

1084

1085

1086

76 Flower, T. G. et al. A helical assembly of human ESCRT-I scaffolds reverse-topology membrane scission. Nature Structural and Molecular Biology 27, 570-580, doi:10.1038/s41594-020-0426-4 (2020).

77 Hierro, A. et al. Structure of the ESCRT-II endosomal trafficking complex. Nature 431, 221-225, doi:10.1038/nature02914 (2004).

78 Teo, H., Perisic, O., González, B. \& Williams, R. L. ESCRT-II, an endosome-associated complex required for protein sorting: crystal structure and interactions with ESCRT-III and membranes. Developmental cell 7, 559-569, doi:10.1016/j.devcel.2004.09.003 (2004).

79 Yang, J. et al. Improved protein structure prediction using predicted interresidue orientations. Proceedings of the National Academy of Sciences of the United States of America 117, 14961503, doi:10.1073/pnas.1914677117 (2020).

$80 \mathrm{Im}, \mathrm{Y}$. J. \& Hurley, J. H. Integrated structural model and membrane targeting mechanism of the human ESCRT-II complex. Developmental cell 14, 902-913, doi:10.1016/j.devcel.2008.04.004 (2008).

81 Bornberg-Bauer, E. \& Albà, M. M. in Current Opinion in Structural Biology Vol. $23 \quad 459-466$ (Elsevier Current Trends, 2013).

82 Copley, S. D. Evolution of new enzymes by gene duplication and divergence. The FEBS Journal 287, 1262-1283, doi:10.1111/febs.15299 (2020).

83 Hong Feng, G., Lih, C.-J. \& Cohen, S. N. TSG101 Protein Steady-State Level Is Regulated Posttranslationally by an Evolutionarily Conserved COOH-Terminal Sequence 1. 1736-1741 (2000).

84 Hug, L. A. et al. Community genomic analyses constrain the distribution of metabolic traits across the Chloroflexi phylum and indicate roles in sediment carbon cycling. Microbiome 1, 22, doi:10.1186/2049-2618-1-22 (2013).

85 Hyatt, D. et al. Prodigal: prokaryotic gene recognition and translation initiation site identification. BMC Bioinformatics 11, 119, doi:10.1186/1471-2105-11-119 (2010).

86 Altschul, S. F. et al. Gapped BLAST and PSI-BLAST: a new generation of protein database search programs. Nucleic Acids Res 25, 3389-3402, doi:10.1093/nar/25.17.3389 (1997).

87 Katoh, K. \& Standley, D. M. MAFFT multiple sequence alignment software version 7: improvements in performance and usability. Mol Biol Evol 30, 772-780, doi:10.1093/molbev/mst010 (2013).

88 Capella-Gutiérrez, S., Silla-Martínez, J. M. \& Gabaldón, T. trimAl: A tool for automated alignment trimming in large-scale phylogenetic analyses. Bioinformatics 25, 1972-1973, doi:10.1093/bioinformatics/btp348 (2009).

89 Minh, B. Q. et al. IQ-TREE 2: New Models and Efficient Methods for Phylogenetic Inference in the Genomic Era. Mol Biol Evol 37, 1530-1534, doi:10.1093/molbev/msaa015 (2020).

90 Hoang, D. T., Chernomor, O., von Haeseler, A., Minh, B. Q. \& Vinh, L. S. UFBoot2: Improving the Ultrafast Bootstrap Approximation. Mol Biol Evol 35, 518-522, doi:10.1093/molbev/msx281 (2018).

91 Jones, P. et al. InterProScan 5: genome-scale protein function classification. Bioinformatics 30, 1236-1240, doi:10.1093/bioinformatics/btu031 (2014).

92 Wickham, $\mathrm{H}$. in Use R!, 1 online resource (XVI, 260 pages 232 illustrations, 140 illustrations in color (Springer International Publishing : Imprint: Springer,, Cham, 2016).

93 Guy, L., Kultima, J. R. \& Andersson, S. G. genoPlotR: comparative gene and genome visualization in R. Bioinformatics 26, 2334-2335, doi:10.1093/bioinformatics/btq413 (2010).

94 Baker, B. J. et al. Genomic inference of the metabolism of cosmopolitan subsurface Archaea, Hadesarchaea. Nat Microbiol 1, 16002, doi:10.1038/nmicrobiol.2016.2 (2016).

95 Geissler, S. et al. The spindle pole body component Spc98p interacts with the gamma-tubulinlike Tub4p of Saccharomyces cerevisiae at the sites of microtubule attachment. The EMBO Journal 15, 3899-3911, doi:10.1002/j.1460-2075.1996.tb00764.x (1996).

96 Gyuris, J., Golemis, E., Chettkov, H. \& Brent, R. Cdil, a Human GI and S Phase Protein Phosphatase That Associates with Cdk2. 791-803 (1993). 
1087

1088

1089

1090

1091

1092

1093

1094

1095

1096

1097

1098

1099

1100

1101

1102

1103

1104

1105

1106

1107

1108

1109

1110

1111

1112

1113

1114

1115

1116

1117

1118

1119

1120

1121

1122

1123

1124

97 Kalyaanamoorthy, S., Minh, B. Q., Wong, T. K. F., von Haeseler, A. \& Jermiin, L. S. ModelFinder: fast model selection for accurate phylogenetic estimates. Nat Methods 14, 587-589, doi:10.1038/nmeth.4285 (2017).

98 Lemoine, F. et al. Renewing Felsenstein's phylogenetic bootstrap in the era of big data. Nature 556, 452-456, doi:10.1038/s41586-018-0043-0 (2018).

99 Wang, H. C., Minh, B. Q., Susko, E. \& Roger, A. J. Modeling Site Heterogeneity with Posterior Mean Site Frequency Profiles Accelerates Accurate Phylogenomic Estimation. Syst Biol 67, 216235, doi:10.1093/sysbio/syx068 (2018).

100 Frey, S. \& Görlich, D. A new set of highly efficient, tag-cleaving proteases for purifying recombinant proteins. Journal of Chromatography A 1337, 95-105, doi:10.1016/j.chroma.2014.02.029 (2014).

101 Liu, L., Spurrier, J., Butt, T. R. \& Strickler, J. E. Enhanced protein expression in the baculovirus/insect cell system using engineered SUMO fusions. Protein Expr Purif 62, 21-28, doi:10.1016/j.pep.2008.07.010 (2008).

102 Micsonai, A. et al. BeStSel: a web server for accurate protein secondary structure prediction and fold recognition from the circular dichroism spectra. Nucleic Acids Res 46, W315-W322, doi:10.1093/nar/gky497 (2018).

103 Frishman, D. \& Argos, P. Knowledge-based protein secondary structure assignment. Proteins 23, 566-579, doi:10.1002/prot.340230412 (1995).

104 Stock, D., Perisic, O. \& Lowe, J. Robotic nanolitre protein crystallisation at the MRC Laboratory of Molecular Biology. Prog Biophys Mol Biol 88, 311-327, doi:10.1016/j.pbiomolbio.2004.07.009 (2005).

105 Gorrec, F. The MORPHEUS protein crystallization screen. Journal of Applied Crystallography 42, 1035-1042, doi:10.1107/S0021889809042022 (2009).

106 Winn, M. D. et al. Overview of the CCP4 suite and current developments. Acta Crystallogr D Biol Crystallogr 67, 235-242, doi:10.1107/S0907444910045749 (2011).

107 Long, F., Vagin, A. A., Young, P. \& Murshudov, G. N. in Acta Crystallographica Section D: Biological Crystallography.1 edn 125-132 (International Union of Crystallography).

108 Emsley, P., Lohkamp, B., Scott, W. G. \& Cowtan, K. Features and development of Coot. Acta Crystallographica Section D: Biological Crystallography 66, 486-501, doi:10.1107/S0907444910007493 (2010).

109 Chen, V. B. et al. MolProbity: All-atom structure validation for macromolecular crystallography. Acta Crystallographica Section D: Biological Crystallography 66, 12-21, doi:10.1107/S0907444909042073 (2010).

110 Götze, M. et al. StavroX-A software for analyzing crosslinked products in protein interaction studies. Journal of the American Society for Mass Spectrometry 23, 76-87, doi:10.1007/s13361011-0261-2 (2012). 
B

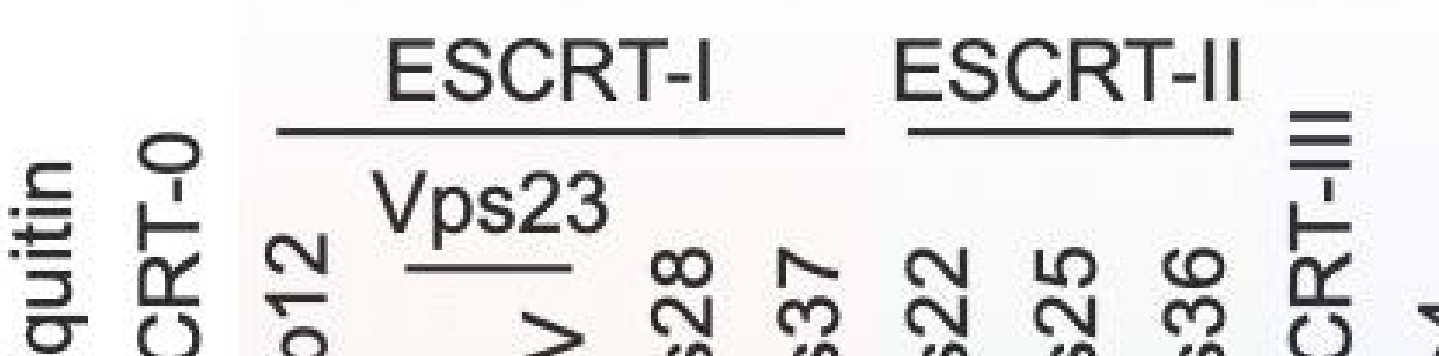

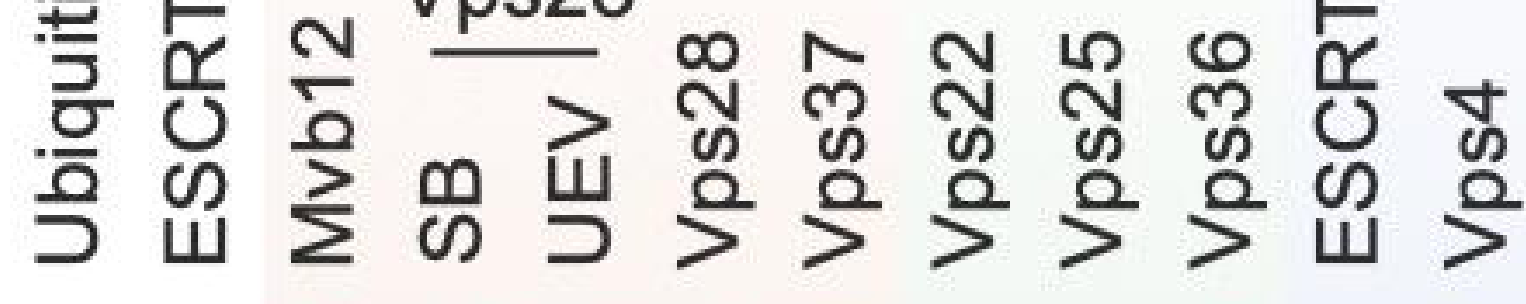

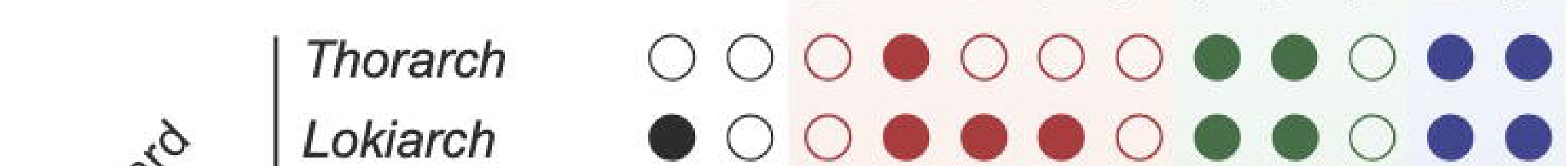
$p^{9^{9^{20^{8}}}}$ OdinLCB4 ๑ ○

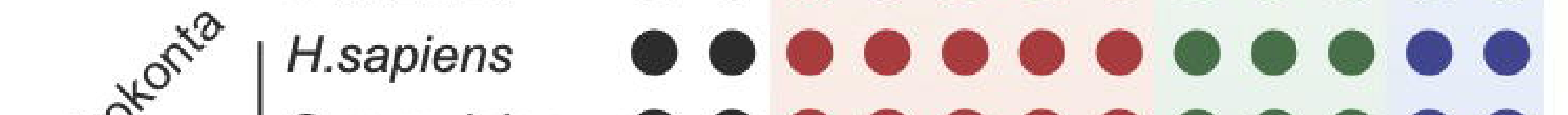
$\mathrm{O}^{\mathrm{sit}}$ a C.neoformans $\bullet \bigcirc$

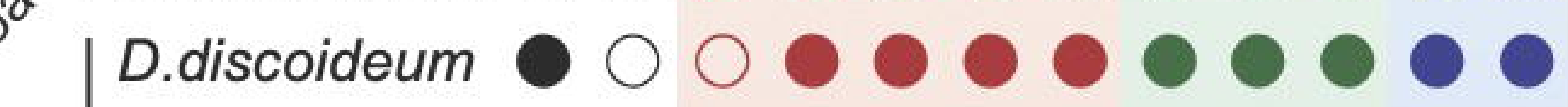

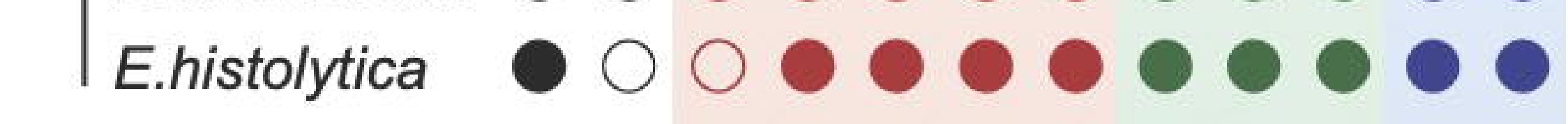
$\gamma^{\curvearrowright} \mid$ A.thaliana $\bullet \bigcirc \bigcirc \bullet \bullet \bullet \bullet \bullet \bullet \bullet \bullet \bullet$ C.rainhardtii $\bullet \bigcirc \bigcirc \bullet \bullet \bullet \bullet \bullet \bullet \bullet \bullet \bullet$ O.tauri $\quad \circ \bigcirc$ C.merolae $\bigcirc \bigcirc \bullet \bullet \bullet \bigcirc \bullet \bullet \bullet \bullet \bullet$ P.falciparum $\bullet \bigcirc \bigcirc \bigcirc \bigcirc \bigcirc \bigcirc \bigcirc \bigcirc \bigcirc \bullet \bullet$ T.gondii $\bullet \bigcirc \bigcirc \bigcirc \bigcirc \bigcirc \bigcirc \bigcirc \bigcirc \bigcirc \bullet \bullet$

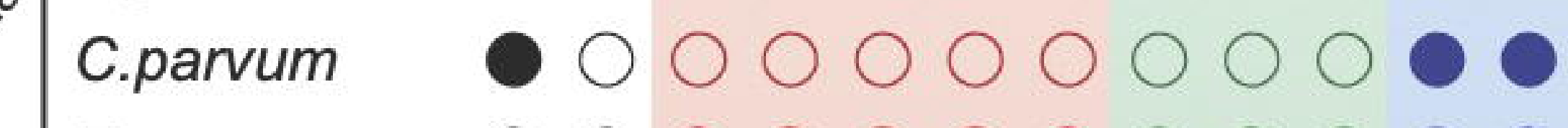
T.parva $\quad 0 \bigcirc \bigcirc \bullet \bullet \bigcirc \bullet \bullet \bullet \bullet \bullet$ T.thermophila $\bigcirc \bigcirc \bullet \bullet \bigcirc \bullet \bullet \bullet \bullet \bullet$ P.ramorum $\bullet \bigcirc \bigcirc \bullet \bullet \bullet \bullet \bullet \bullet \bullet \bullet \bullet$ T.pseudonana $\bigcirc \bigcirc \bullet \bullet \bullet \bullet \bullet \bullet \bullet \bullet \bullet$ T.brucei $\quad \bullet \bigcirc \bigcirc \bullet \bullet \bullet \bullet \bullet \bullet \bullet \bullet \bullet$

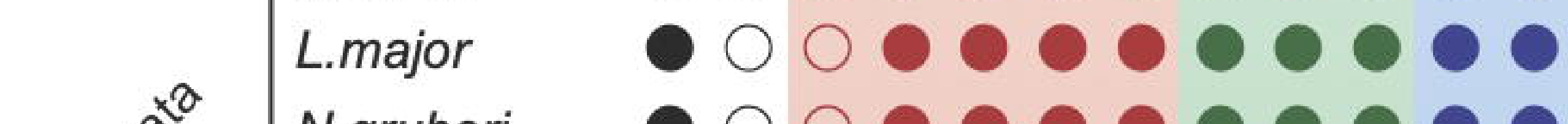
$\left\langle t^{0^{20}}\right.$ G.intestinalis $\bigcirc \bigcirc \bigcirc \bigcirc \bigcirc \bigcirc$

C

Heimdallarchaeote AB_125

Heimdallarchaeote B3_Heim

Helarchaeote Hel_GB_B

Lokiarchaeote GC14_75

Prometheoarchaeum syntrophicum

Odinarchaeote LCB_4

Thorarchaeote AB_25

Thorarchaeote L.E.AR.5
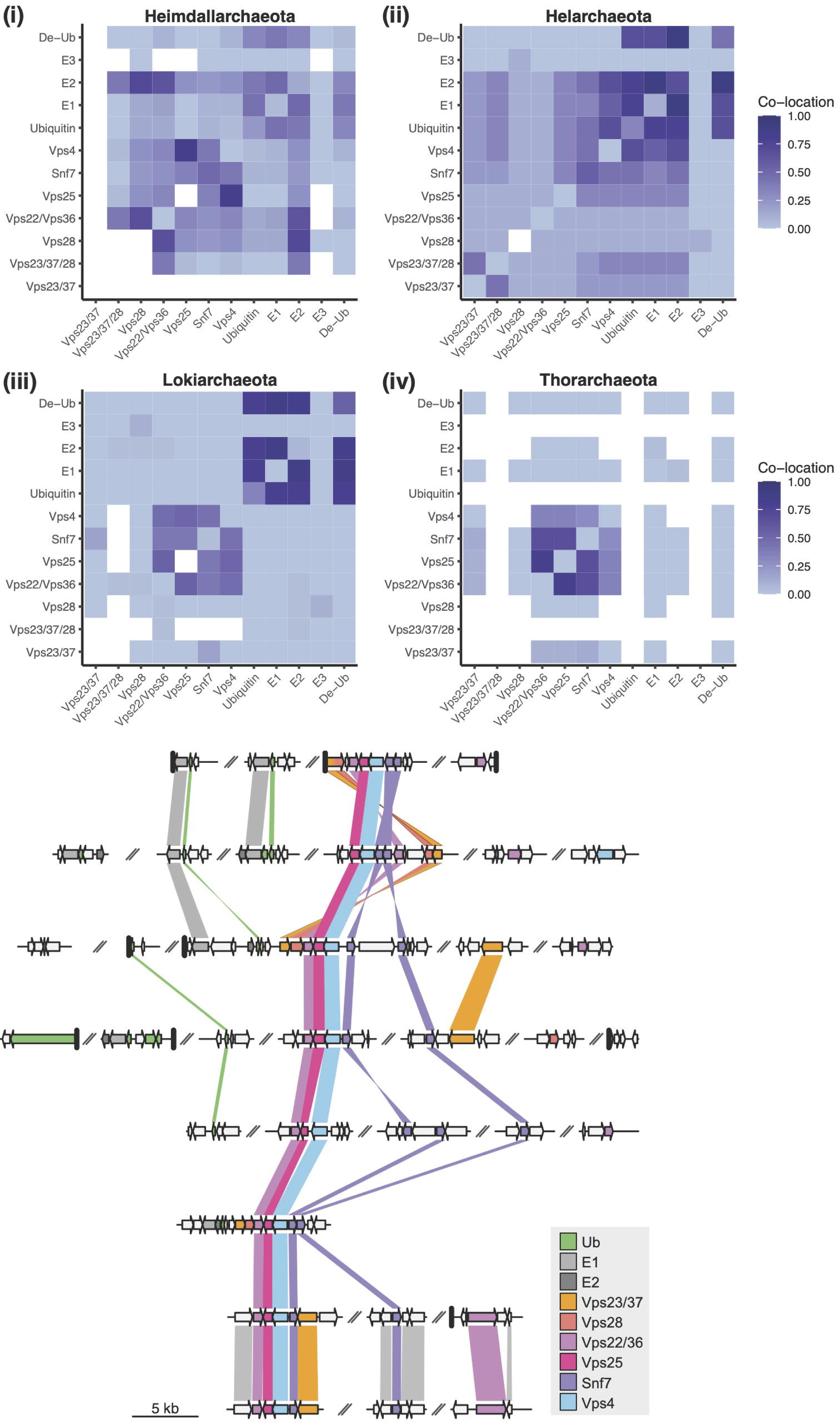

"

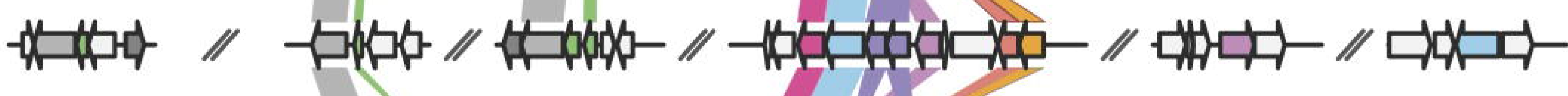

-4

一

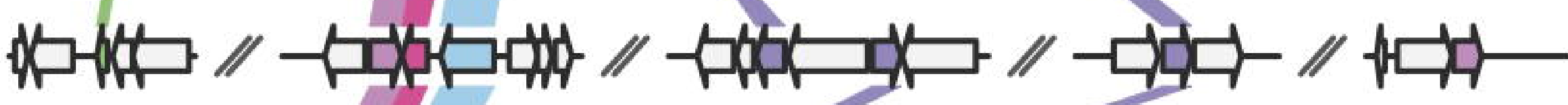

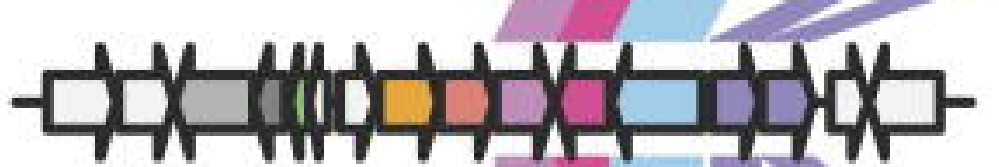

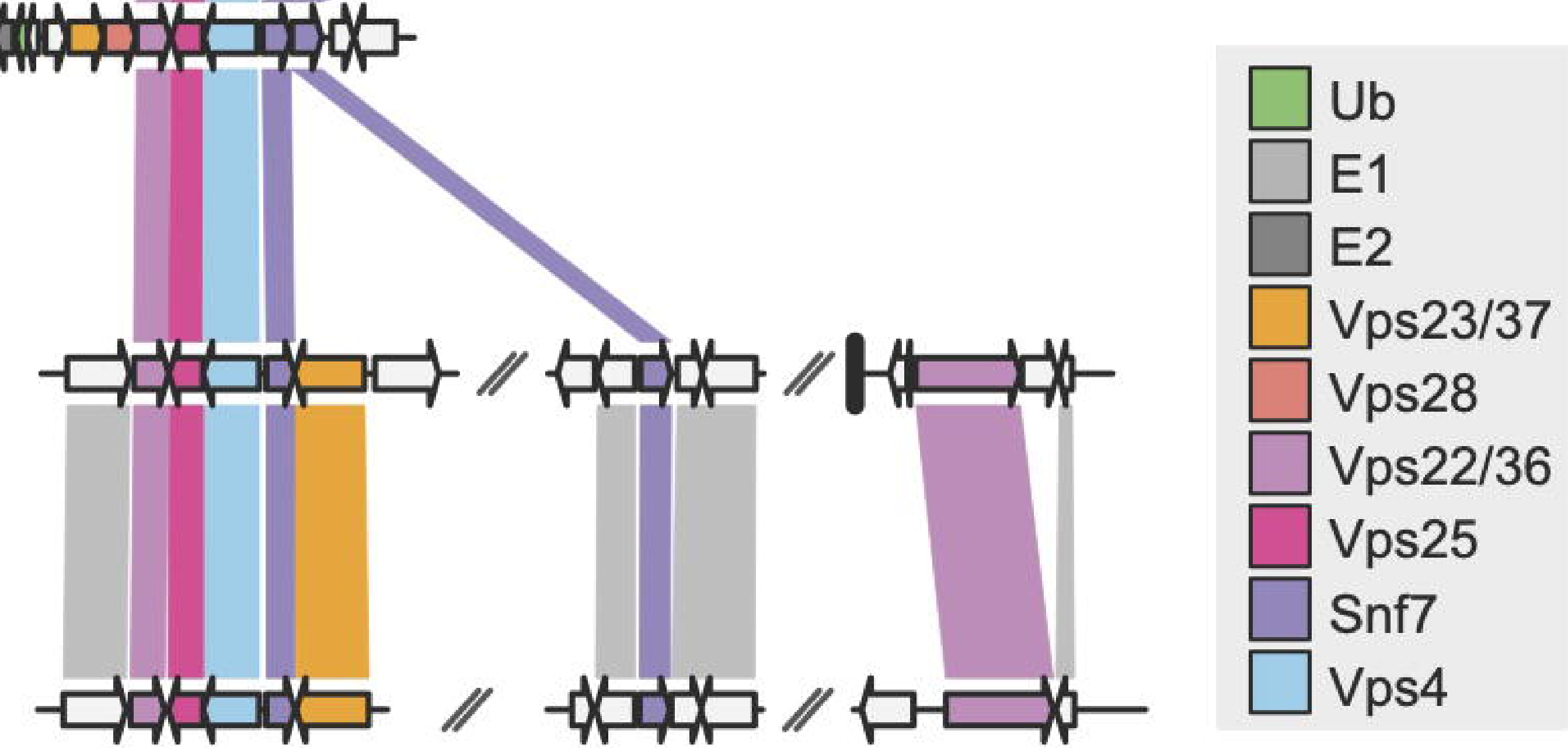

E

D

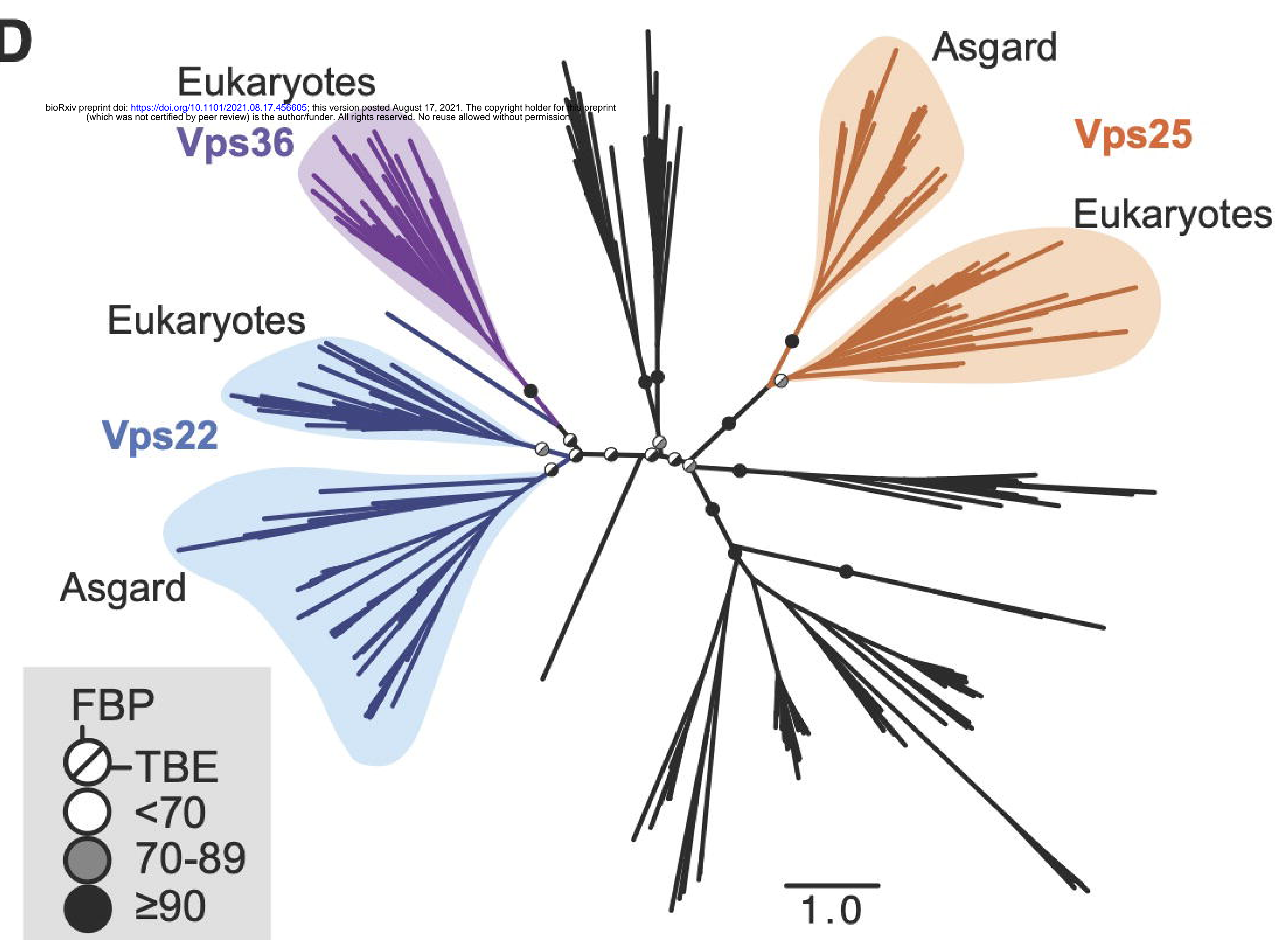

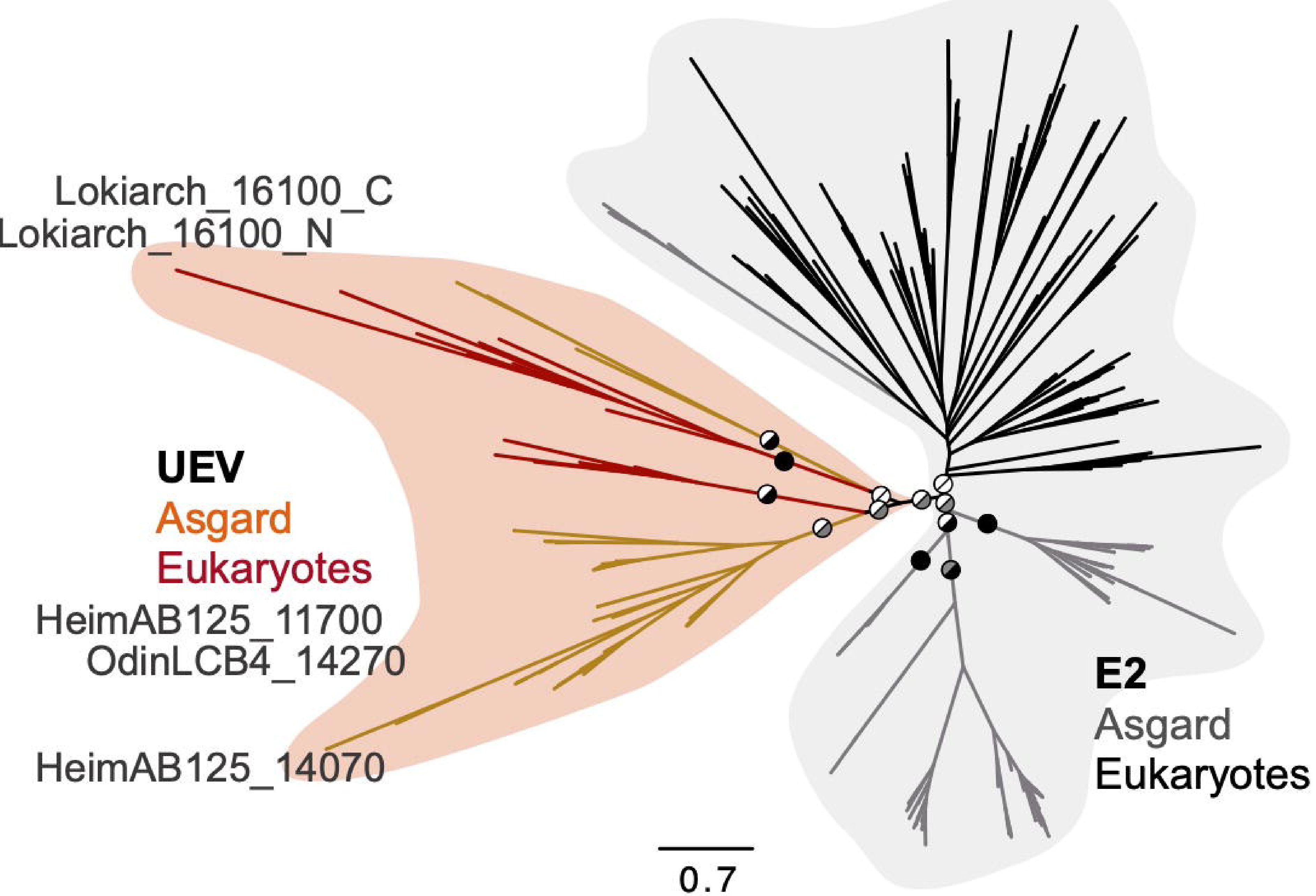


A

\begin{tabular}{rr|}
\hline UEV & VPS28 \\
\hline & 469 aa \\
\hline & 469 aa \\
\hline
\end{tabular}
130 aа

B

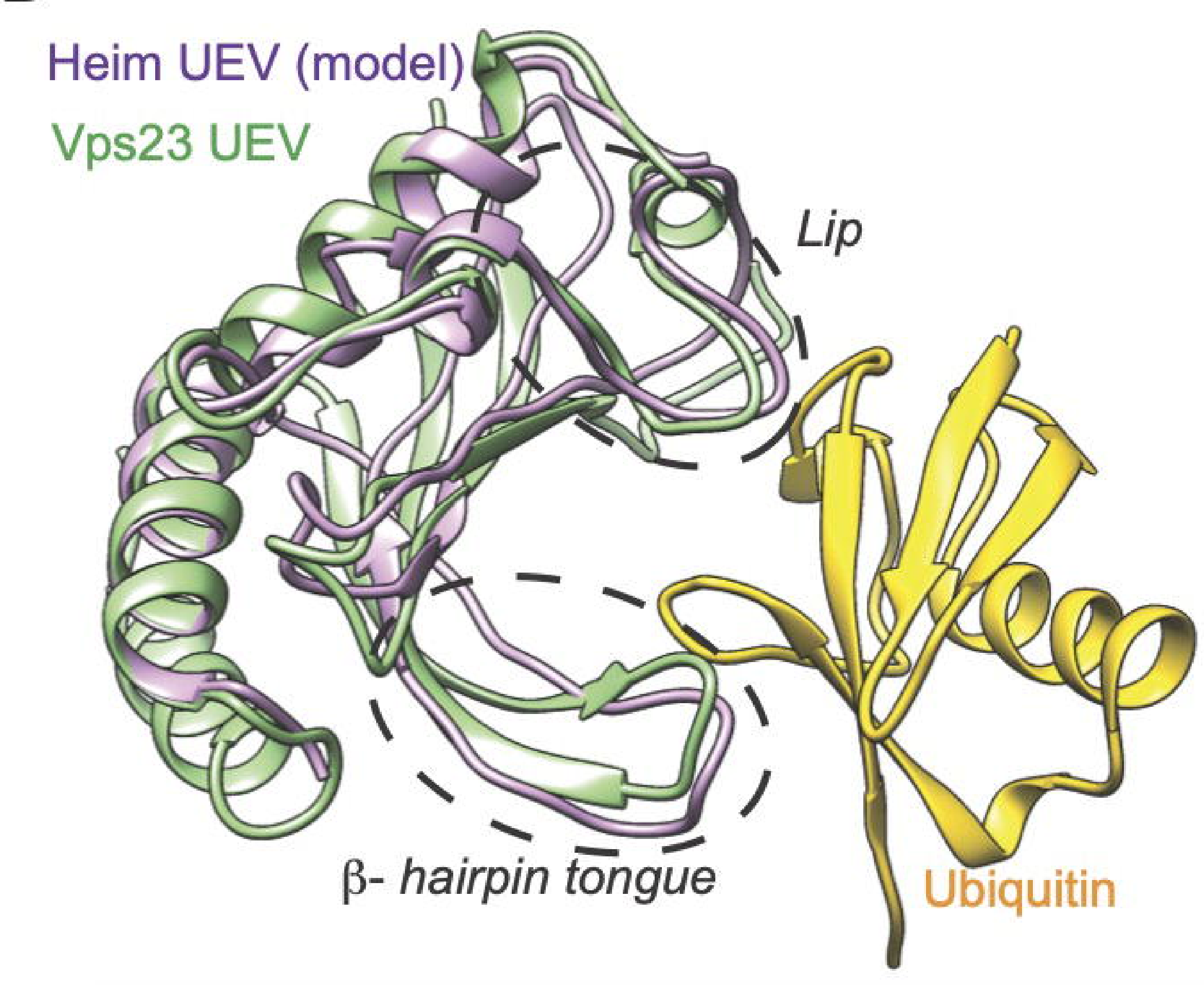

C

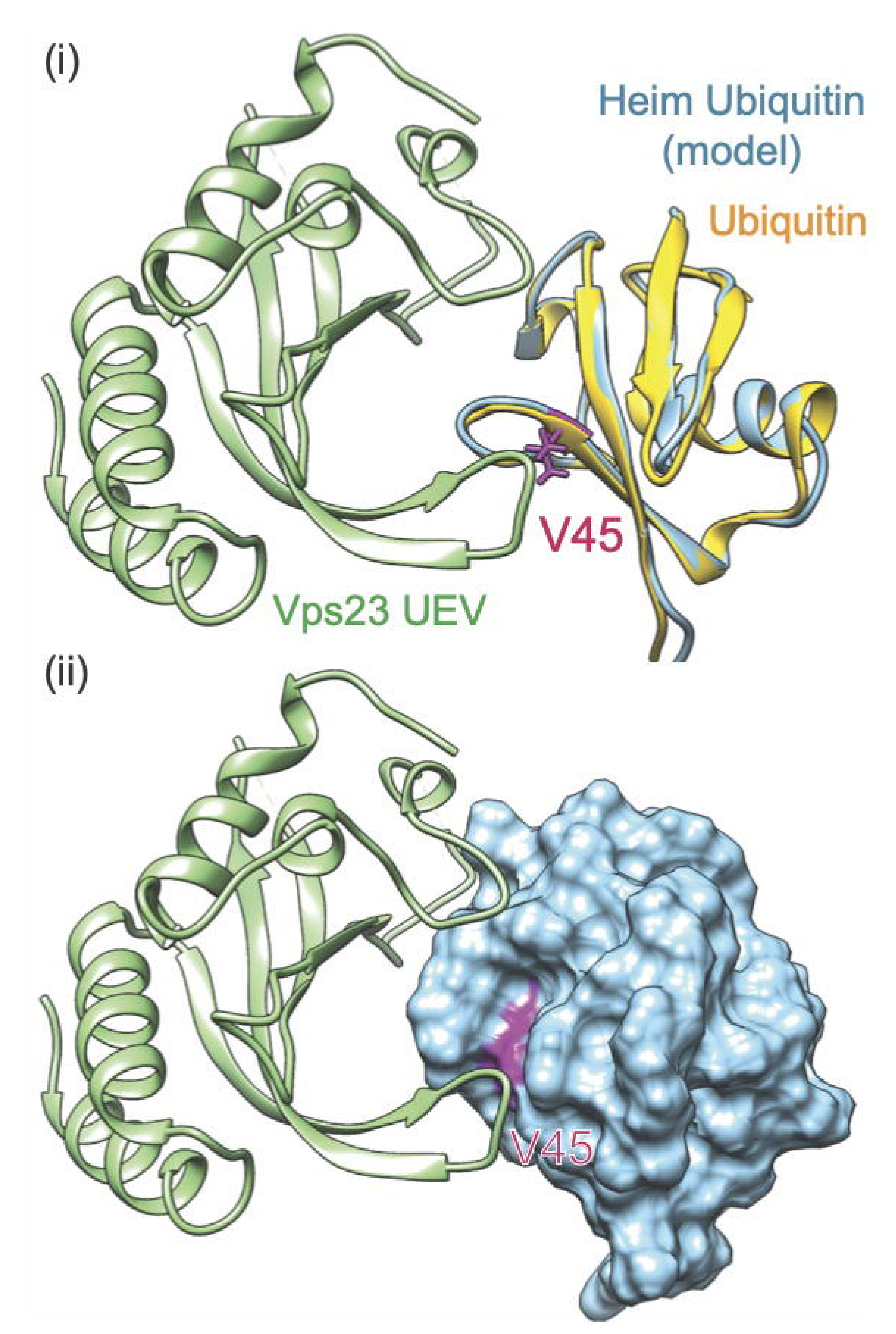

D

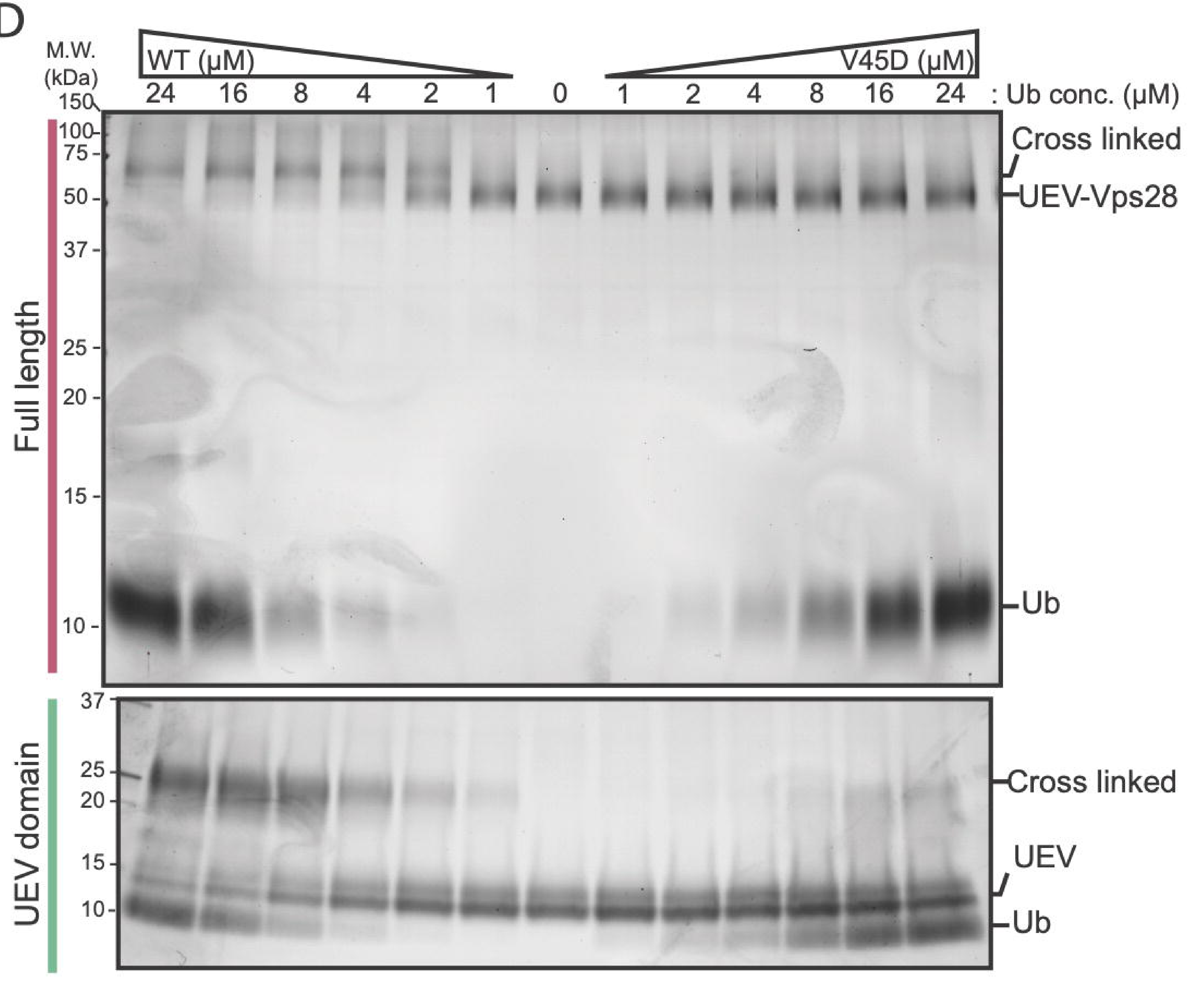

E
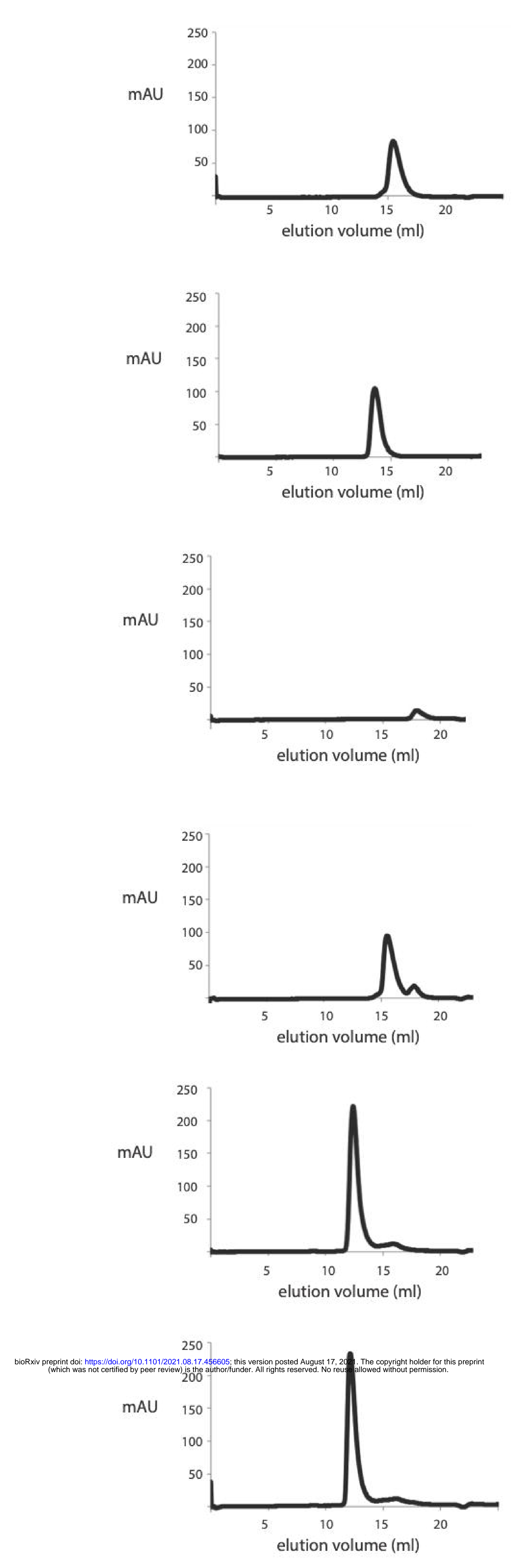

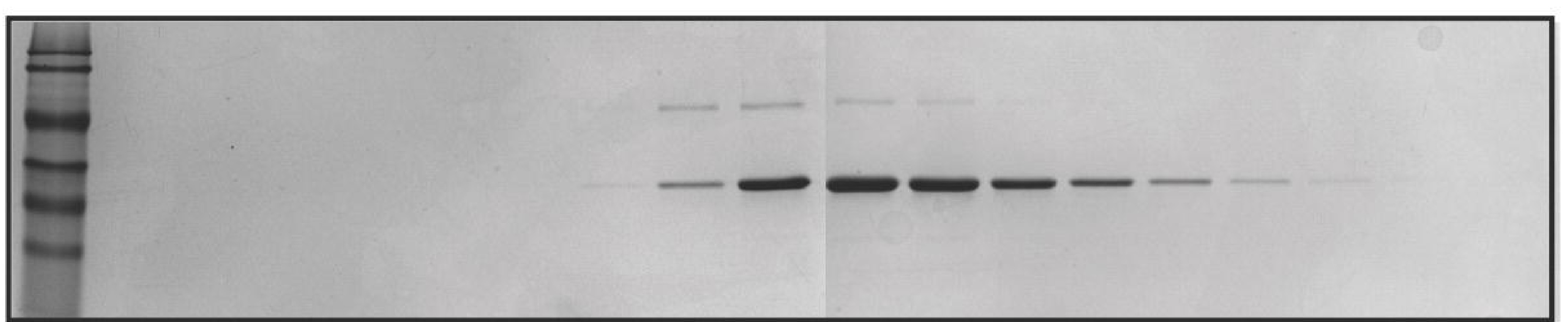

Odinarchaeota ESCRT-I proteins

Vps28 only

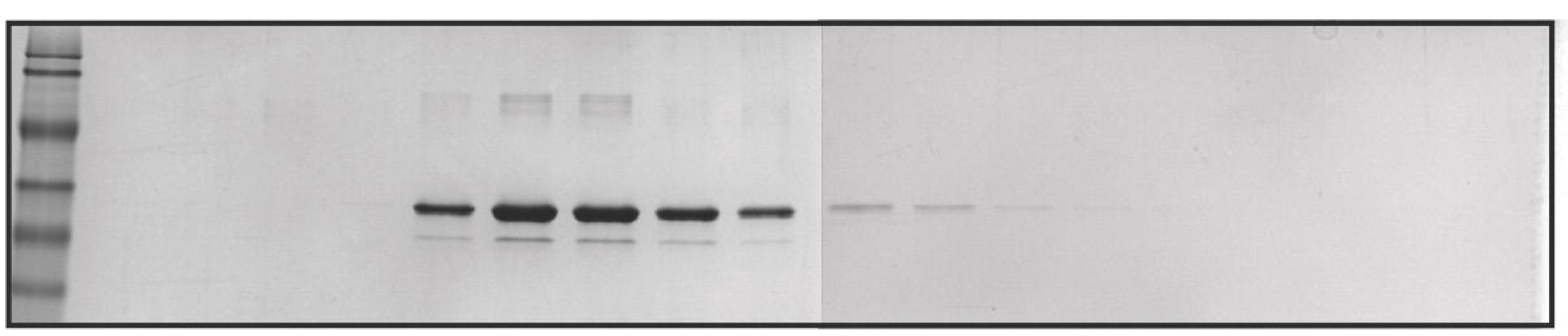

Vps23(TSG101) only

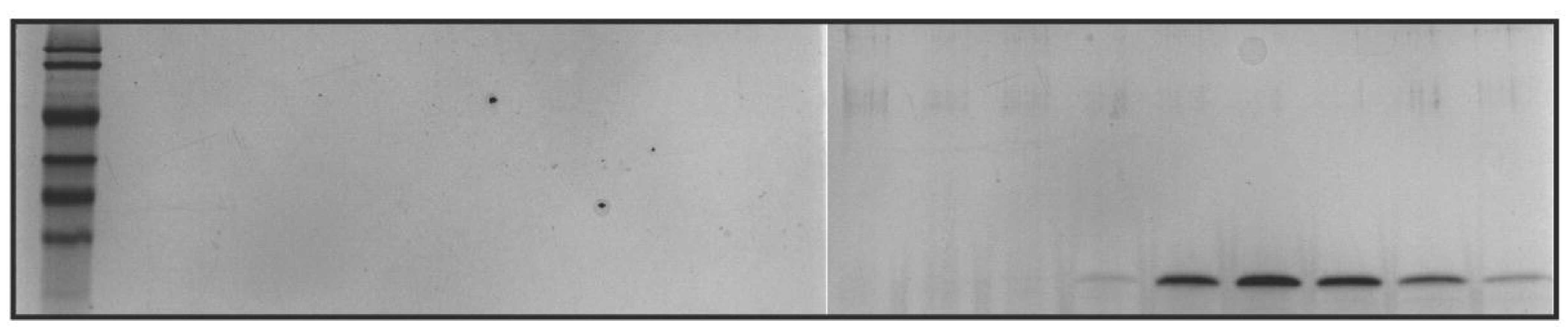

ubiquitin only

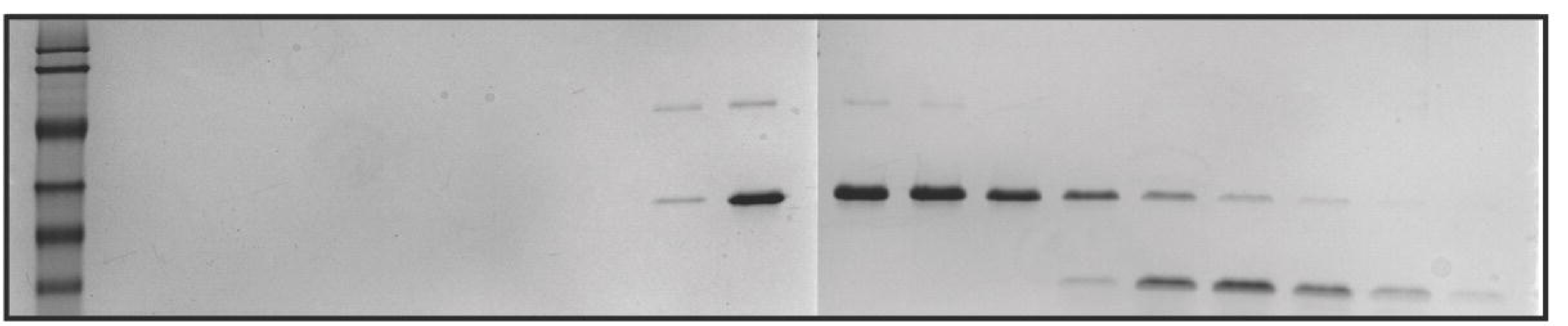

Vps28

plus ubiquitin

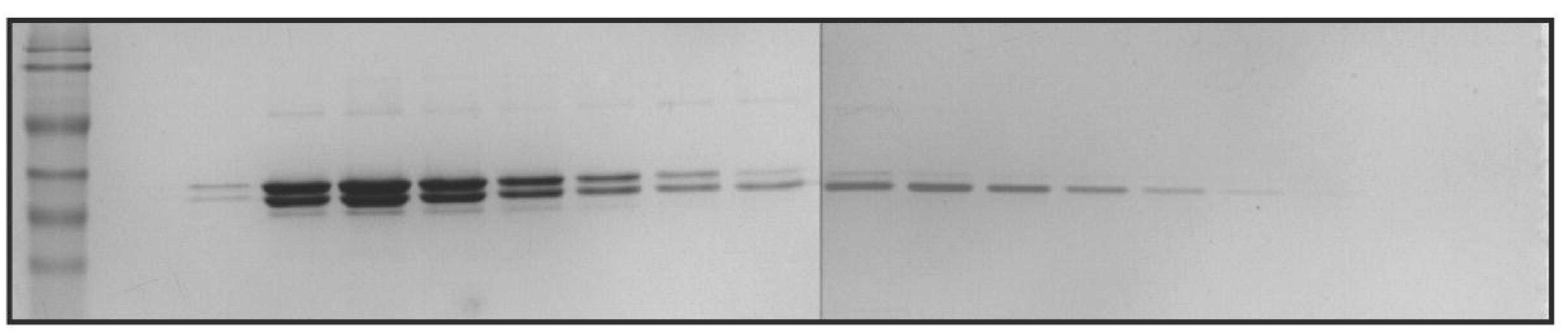

Vps23(TSG101)/Nps28 complex

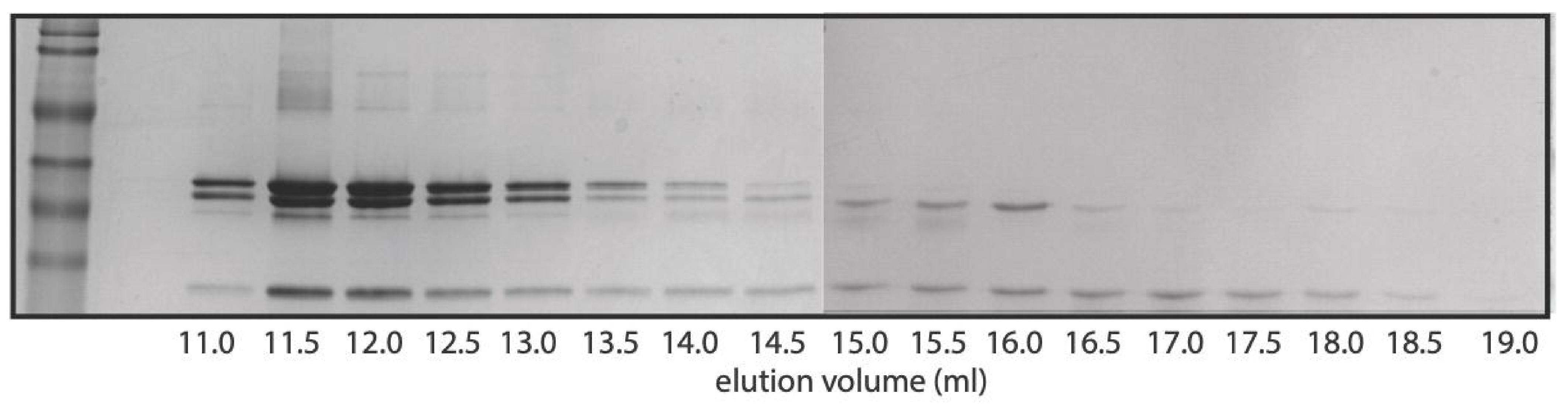

ubiquitinVps23(TSG101) Nps28 complex

$$
\begin{array}{ccccc}
\uparrow & \uparrow & \uparrow & \uparrow & \uparrow \\
12.4 \mathrm{ml} & 13.6 \mathrm{ml} & 14.6 \mathrm{ml} & 17.4 \mathrm{ml} & 18.9 \mathrm{ml} \\
\beta \text {-amylase } & \text { Alcohol } & \text { BSA } & \text { Carbonic } & \text { Cytochrome c } \\
(200 \mathrm{kDa}) & \text { dehydrogenase } & (66 \mathrm{kDa}) & \text { anhydrase } & (12.4 \mathrm{kDa}) \\
& (150 \mathrm{kDa}) & (29 \mathrm{kDa}) &
\end{array}
$$



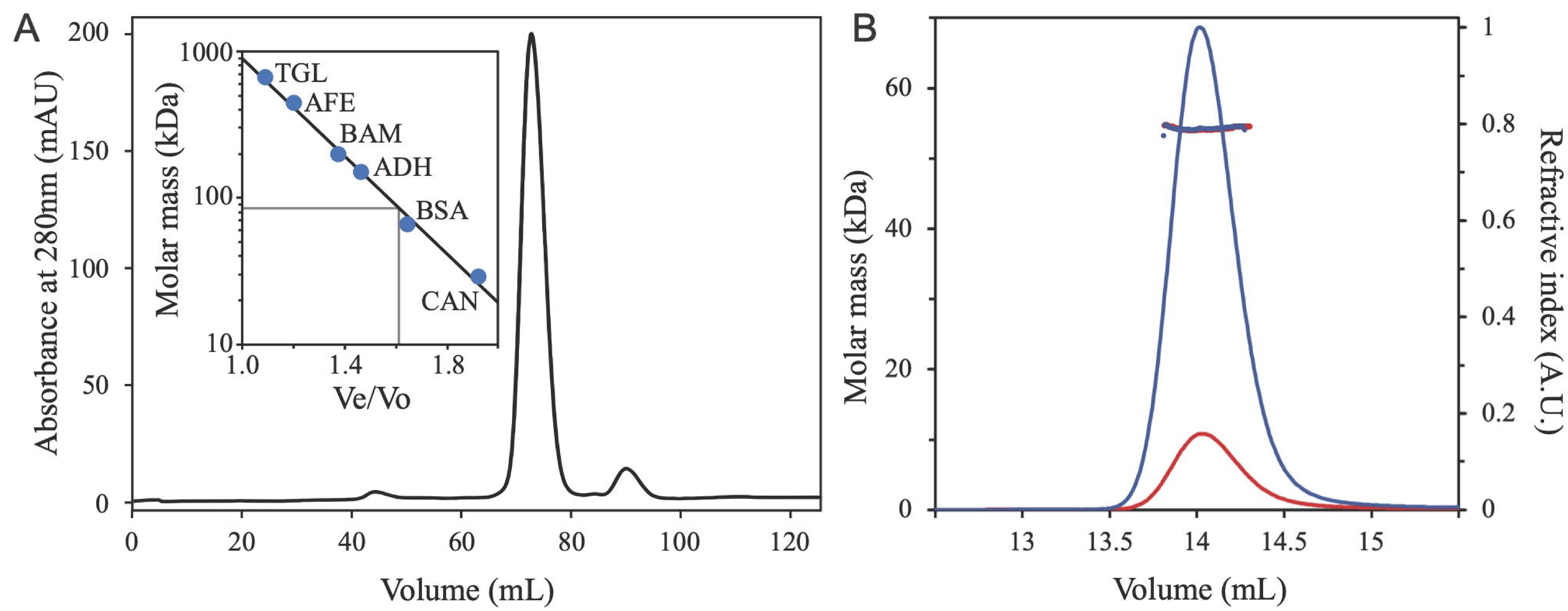

C
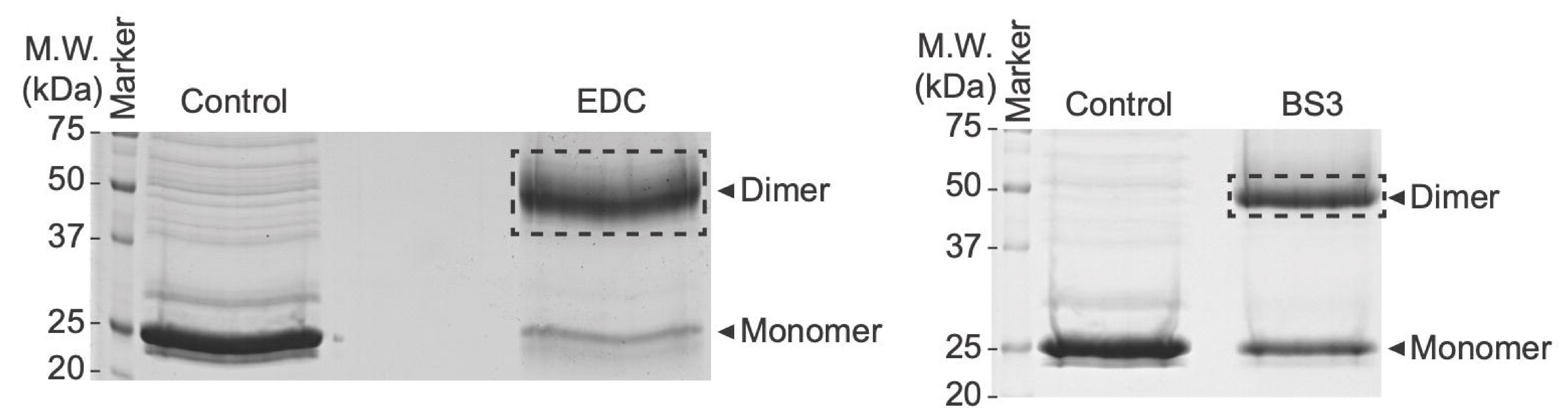

D

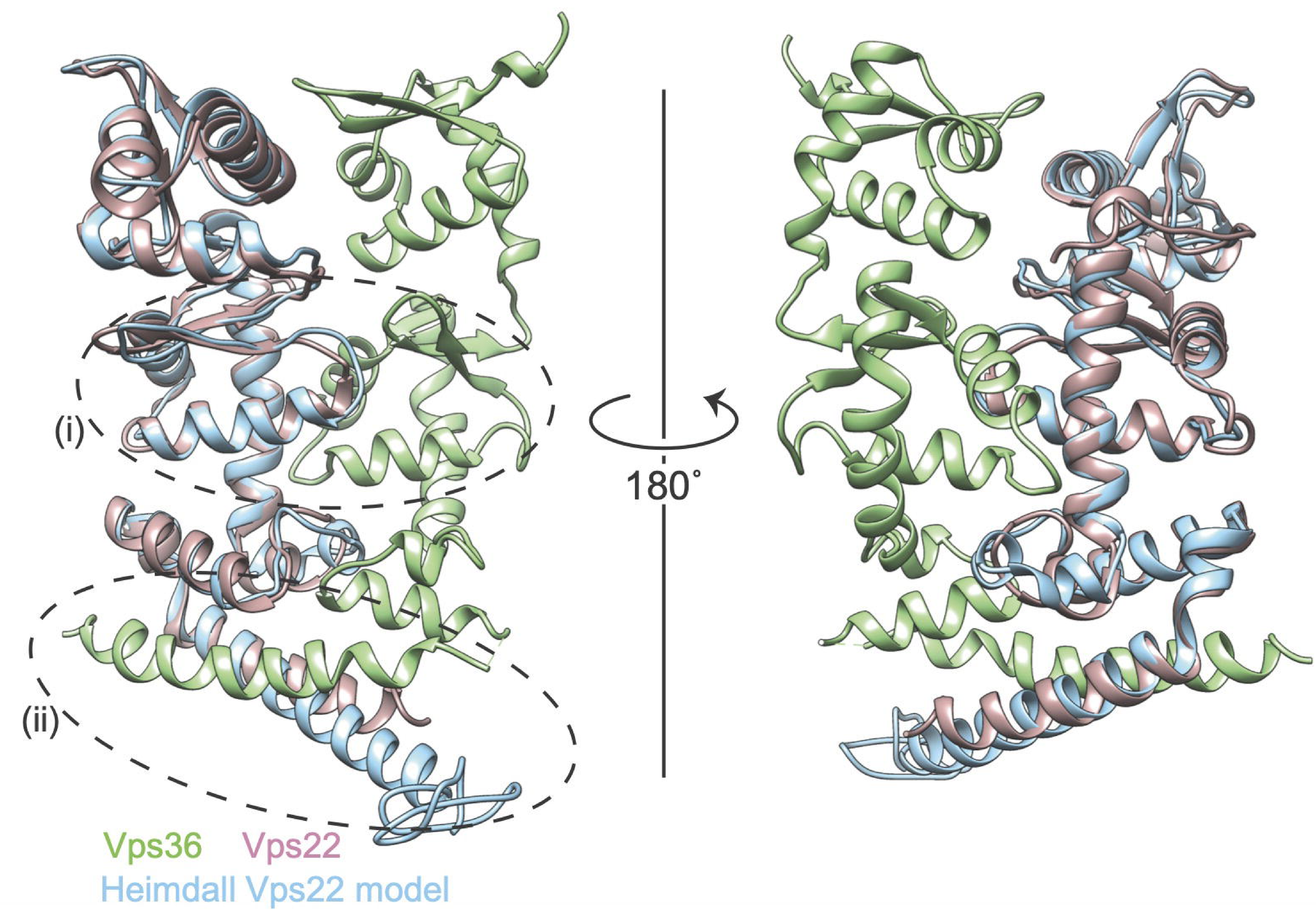

$E$

(i)

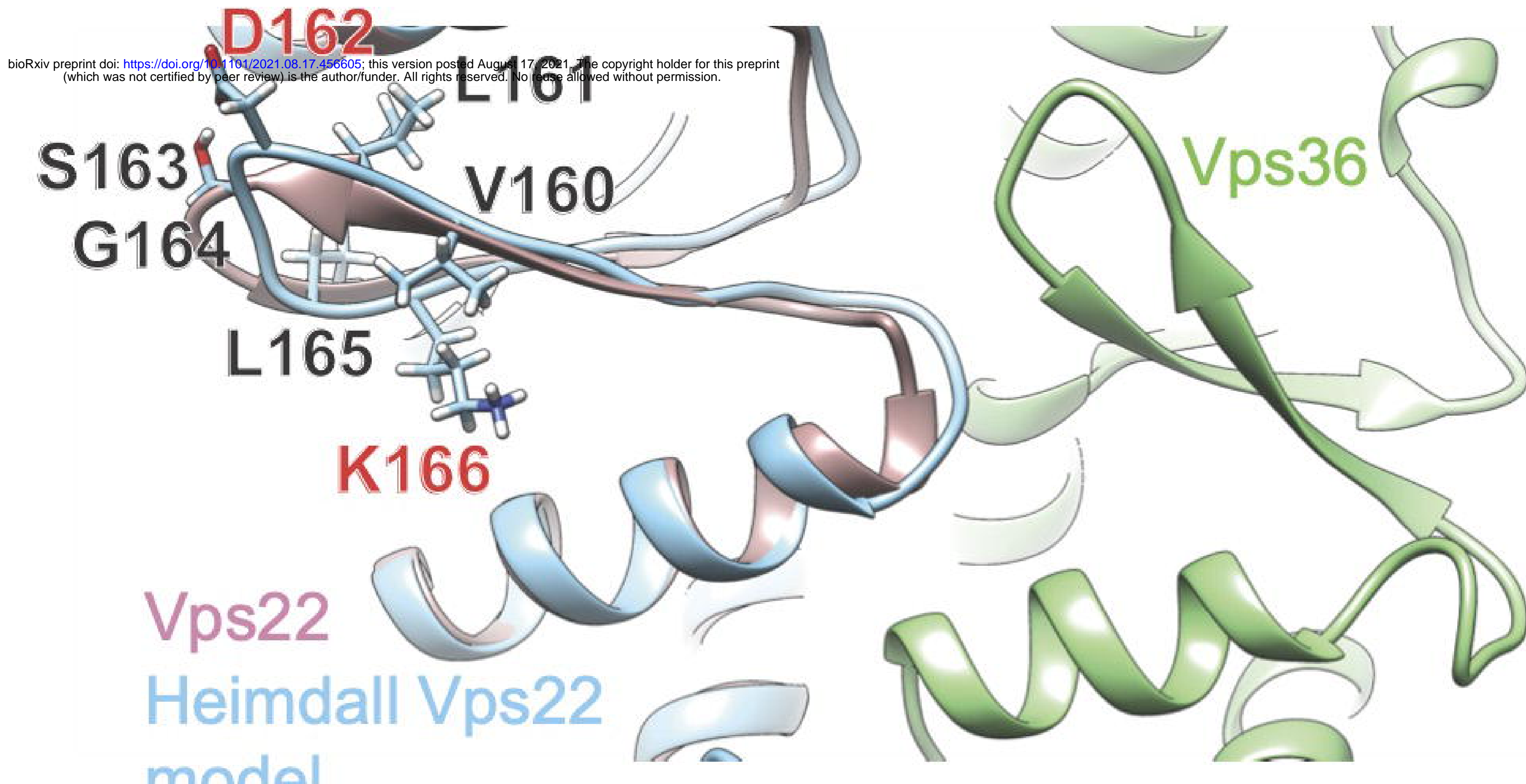
model

alpha: 160-VLDSGLK-166

beta: 160-VLDSGLK-166 (ii)

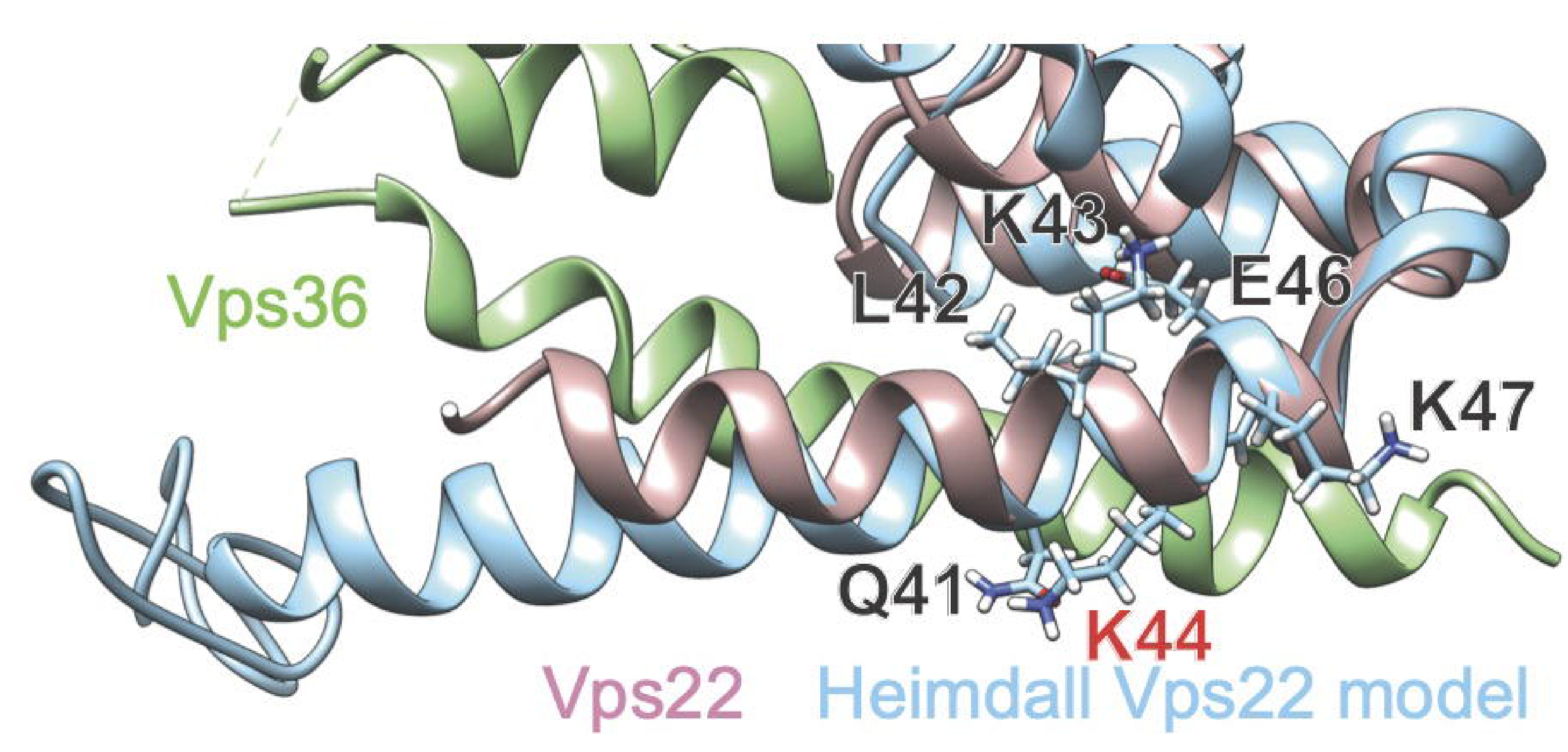

alpha: 41-QLKK-44

beta: 
A

Archaeal Vps25

(N-terminus, blue;

C-terminus, red)

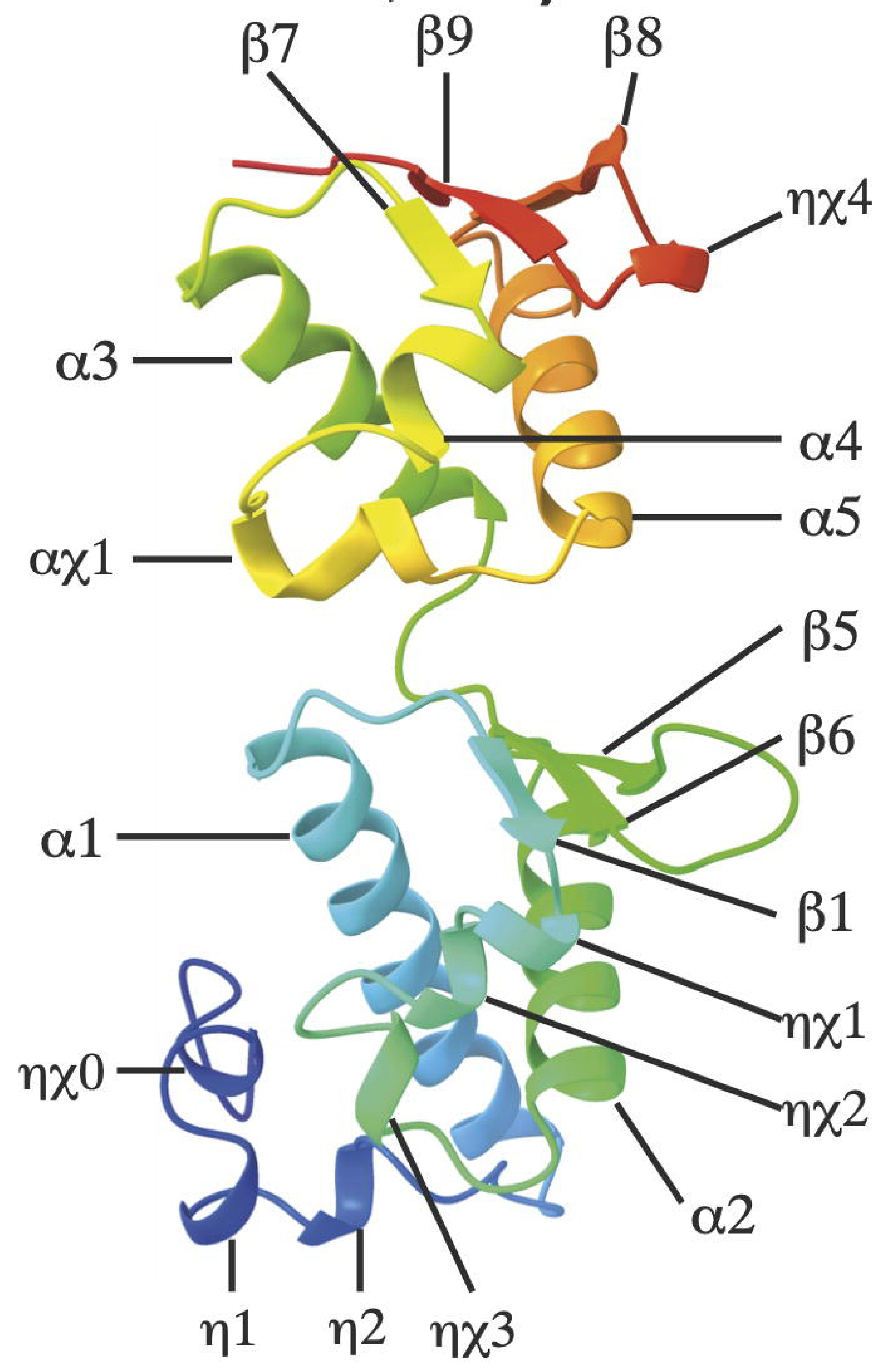

B

Superposition of the $\mathrm{n}$ - (blue) and c-terminal (pink) archaeal Vps25 wH domains
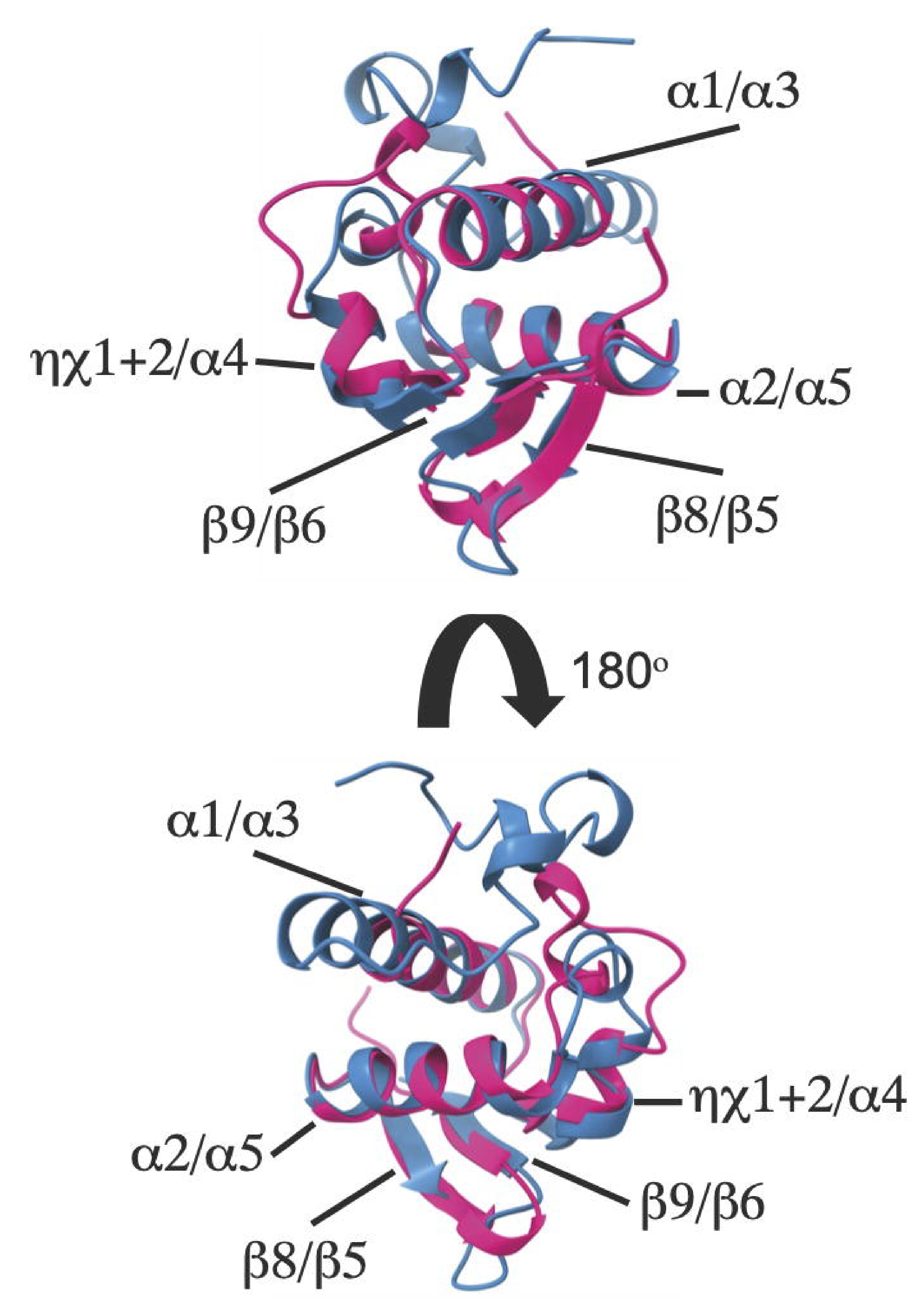

C

Comparison of archaeal Vps25 (red) with

Vps25 from S. cerevisiae (green)

(1XB4) and $H$. sapiens (blue) (2ZME)
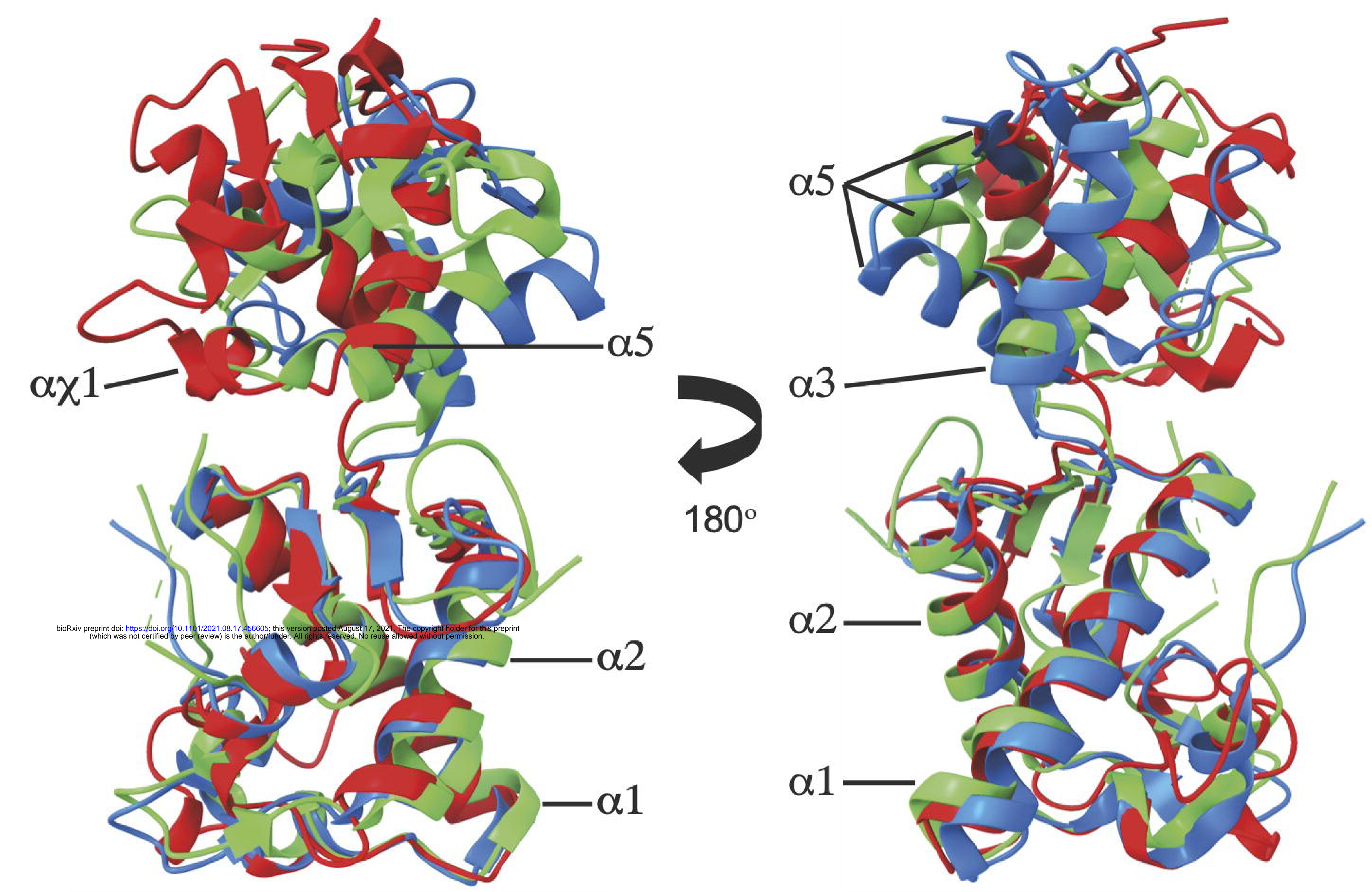


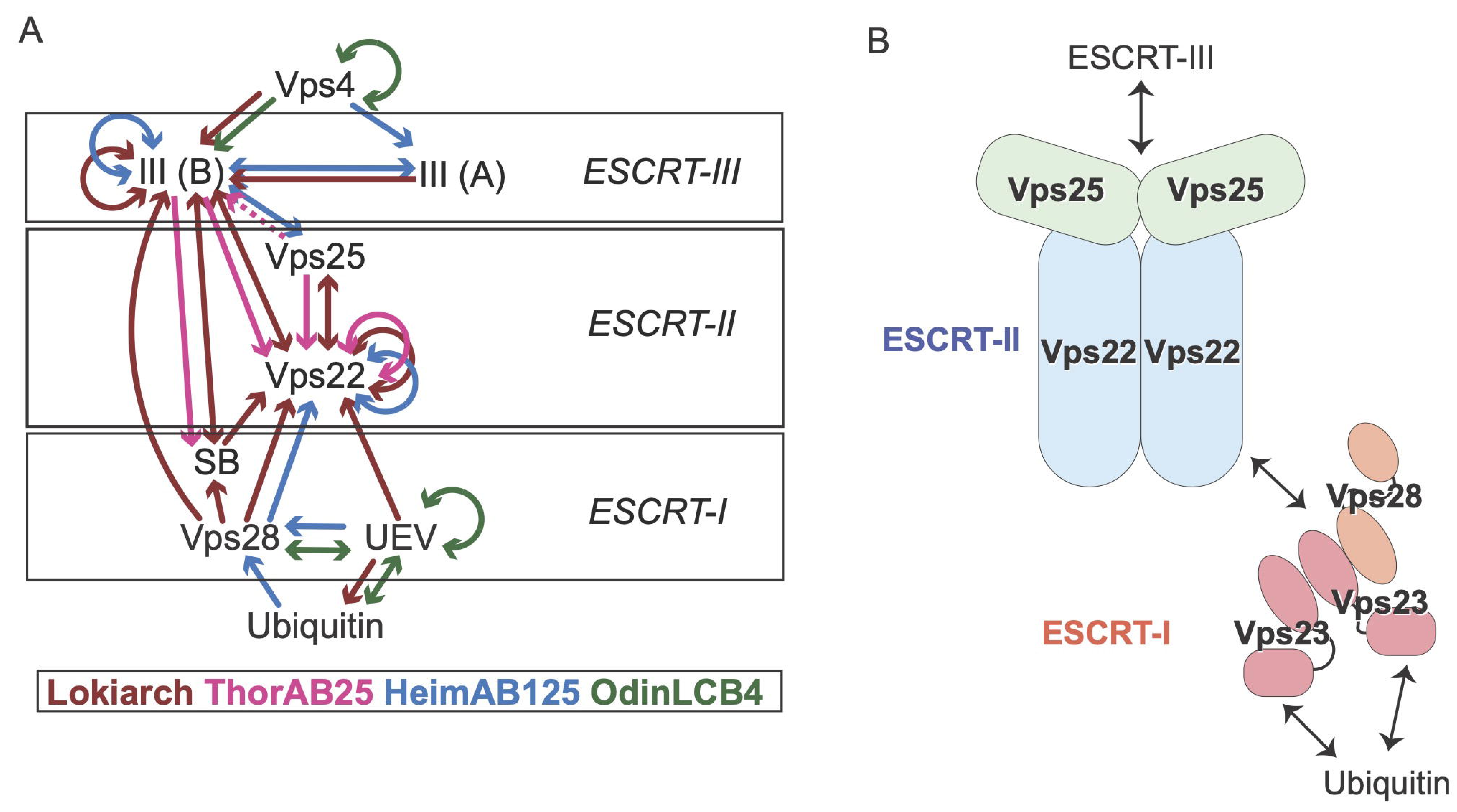

